Federal Reserve Bank of Minneapolis

Research Department Staff Report 346

September 2004

\title{
A Unified Framework for Monetary Theory and Policy Analysis*
}

\author{
Ricardo Lagos \\ New York University \\ and Federal Reserve Bank of Minneapolis \\ Randall Wright \\ University of Pennsylvania \\ and Federal Reserve Bank of Cleveland
}

\begin{abstract}
Search-theoretic models of monetary exchange are based on explicit descriptions of the frictions that make money essential. However, tractable versions of these models typically need strong assumptions that make them ill-suited for studying monetary policy. We propose a framework based on explicit micro foundations within which macro policy can be analyzed. The model is both analytically tractable and amenable to quantitative analysis. We demonstrate this by using it to estimate the welfare cost of inflation. We find much higher costs than the previous literature: our model predicts that going from $10 \%$ to $0 \%$ inflation can be worth between $3 \%$ and $5 \%$ of consumption.
\end{abstract}

*First version: May 2000. Many people provided extremely helpful input to this project, including S.B. Aruoba, A. Berentsen, V.V. Chari, L. Christiano, N. Kocherlakota, G. Rocheteau, S. Shi, C. Waller, and N. Wallace. Financial support from the C.V. Starr Center for Applied Economics at NYU, STICERD at the LSE, the NSF, and the Federal Reserve Banks of Cleveland and Minneapolis is acknowledged. The views expressed herein are those of the authors and not necessarily those of the Federal Reserve Bank of Cleveland, the Federal Reserve Bank of Minneapolis, or the Federal Reserve System. 


\section{Introduction}

Existing monetary models in macroeconomics are reduced-form models. By this we mean they make assumptions, such as putting money in the utility function or imposing cash-in-advance constraints, that are meant to stand in for some role of money that is not made explicit - say, that it helps overcome spatial, temporal, or informational frictions. There are models that provide micro foundations for monetary economics using search theory, based on explicit descriptions of specialization, the pattern of meetings, the information structure, and so on. This framework can be used to address issues such as: what types of frictions make the use of money an equilibrium or efficient arrangement; which objects endogenously end up serving as media of exchange; or how do different regimes (e.g., commodity versus fiat money, or one currency versus two) lead to different outcomes. But these models are ill suited for the analysis of monetary policy as it is usually formulated. The reason is that the typical search-based model becomes intractable without very strong assumptions, including extreme restrictions on how much money agents can hold. Therefore, if one wants to study policy there is little choice but to resort to the reduced-form approach. ${ }^{1}$

In this paper we propose a framework that attempts to bridge this gap: it is based explicitly on microeconomic frictions and can be used to study a range of macroeconomic policies. As an application we carry out a quantitative analysis of the welfare cost of inflation. This exercise clearly illustrates

\footnotetext{
${ }^{1}$ In terms of the literature, examples of reduced-form models include Lucas and Stokey (1983, 1987), Cooley and Hansen (1989, 1991), and Christiano et al. (1997); see Walsh (1998) for more references. Examples in the search literature with strong assumptions on money holdings include Kiyotaki and Wright $(1989,1991)$, Shi (1995), Trejos and Wright (1995), Kocherlakota (1998), and Wallace (2001). Other search models are discussed below.
} 
that, besides being analytically tractable, the model can also be realistically parametrized. We find much higher welfare costs than the previous literature: our model predicts that going from $10 \%$ to $0 \%$ inflation can be worth between $3 \%$ and $5 \%$ of consumption.

There are previous attempts to study search models without severe restrictions on money holdings, $m$. Trejos and Wright (1995) present a version of their model where agents can hold any $m \in \mathbb{R}_{+}$, but cannot solve it and end up resorting to $m \in\{0,1\}$. The model with $m \in \mathbb{R}_{+}$is studied numerically by Molico (1999). Although his findings are interesting, the framework is very complicated: basically no analytic results are available and even numerical analysis is difficult. What makes the problem complicated is the endogenous distribution of money holdings, $F(m)$. Our model integrates decentralized trade as in search theory with periodic access to centralized markets. For a certain class of preferences, we find that all agents in the centralized market choose the same money balances, which renders $F$ degenerate in the decentralized market. ${ }^{2}$ The model in Shi (1997) is related to our framework and also gets $F$ degenerate, although by different means. We will compare these different approaches in considerable detail below, after describing how our model works.

The rest of the paper is organized as follows. Section 2 describes the environment. Section 3 defines equilibrium and derives some basic results.

\footnotetext{
${ }^{2}$ The relevant class of preferences displays a certain quasi-linearity. Intuitively, quasilinearity implies the amount of money you take to the market today does not depend on how much you came home with last night. We suggest below that this may not be a bad empirical approximation, but the main advantage is really analytic convenience. Another argument for quasi-linear preferences, however, is that they are used in many other macroeconomic applications, including much real business cycle theory and reducedform monetary economics; examples include Hansen (1985), Rogerson (1988), Cooley and Hansen $(1989,1991)$, and Christiano and Eichenbaum (1992).
} 
Section 4 discusses the related literature, including Shi (1997). Section 5 introduces monetary policy considerations. Section 6 uses a calibrated version to quantify the welfare cost of inflation. Section 7 concludes. The Appendix contains many technical results.

\section{The Model}

Time is discrete. There is a $[0,1]$ continuum of agents who live forever with discount factor $\beta \in(0,1)$. Each period is divided into two subperiods, day and night. Agents consume and supply labor in both subperiods. In general, preferences are $\mathcal{U}(x, h, X, H)$, where $x$ and $h(X$ and $H)$ are consumption and labor during the day (night). It is important for tractability that $\mathcal{U}$ is linear in either $X$ or $H$. We assume

$$
\mathcal{U}(x, h, X, H)=u(x)-c(h)+U(X)-A H,
$$

although separability across $(x, h, X)$ is not critical and is made merely to ease the presentation. Assume $u, c$, and $U$ are $C^{n}$ ( $n$ times continuously differentiable) with $n \geq 2$. Also, assume $U^{\prime}>0, U^{\prime \prime} \leq 0, U$ is either unbounded or satisfies a condition in Lemma 6 , and there exists $X^{*} \in(0, \infty)$ such that $U^{\prime}\left(X^{*}\right)=1$ and $U\left(X^{*}\right)>X^{*}$. Also, assume $u(0)=c(0)=0$, $u^{\prime}>0, c^{\prime}>0, u^{\prime \prime}<0, c^{\prime \prime} \geq 0$, and there exists $q^{*} \in(0, \infty)$ such that $u^{\prime}\left(q^{*}\right)=c^{\prime}\left(q^{*}\right)$.

Economic activity differs across the subperiods. As in the typical search model, during the day agents interact in a decentralized market involving anonymous bilateral matching with arrival rate $\alpha$. We assume the day good $x$ comes in many specialized varieties, of which each agent consumes only a subset. An agent can transform labor one for one into a special good 
that he does not consume. This generates a double coincidence problem. In particular, for two agents $i$ and $j$, drawn at random, there are four possible events. The probability that both consume what the other can produce (a double coincidence) is $\delta$. The probability that $i$ consumes what $j$ produces but not vice versa (a single coincidence) is $\sigma$. Symmetrically, the probability $j$ consumes what $i$ produces but not vice versa is $\sigma$. And the probability that neither wants what the other produces is $1-2 \sigma-\delta$, where $2 \sigma \leq 1-\delta .^{3}$ In a single coincidence meeting, if $i$ wants the special good that $j$ produces, we call $i$ the buyer and $j$ the seller.

At night agents trade in a centralized (Walrasian) market. With centralized trade, specialization does not lead to a double coincidence problem, and so it is irrelevant if the night good $X$ comes in many varieties or only one; hence we assume that at night all agents produce and consume a general good, mainly because this allows a closer comparison with standard macro models. Agents at night can transform 1 unit of labor into $w$ units of the general good, where we assume here that $w$ is a technological constant, and be normalized to $w=1$ with no loss in generality. ${ }^{4}$ We assume the general goods produced at night and the special goods produced during the day are nonstorable. There is another object, called money, that is perfectly divisible and can be stored in any quantity $m \geq 0$.

\footnotetext{
${ }^{3}$ This notation captures several specifications in the literature as special cases. In Kiyotaki and Wright (1989) or Aiyagari and Wallace (1991) there are $N$ goods and $N$ types, and type $n$ produces good $n$ and consumes good $n+1, \bmod N$. If $N>2, \sigma=1 / N$ and $\delta=0$, while if $N=2, \delta=1 / 2$ and $\sigma=0$. In Kiyotaki and Wright (1993) the event that $i$ consumes what $j$ produces is independent of the event that $j$ consumes what $i$ produces, and each occurs with probability $x$. Then $\delta=x^{2}$ and $\sigma=x(1-x)$.

${ }^{4}$ Aruoba and Wright (2003) show how to recast things in terms of agents selling labor to neoclassical firms for $w$ units of purchasing power, where $w$ is determined to clear the labor market; this changes nothing of substance.
} 


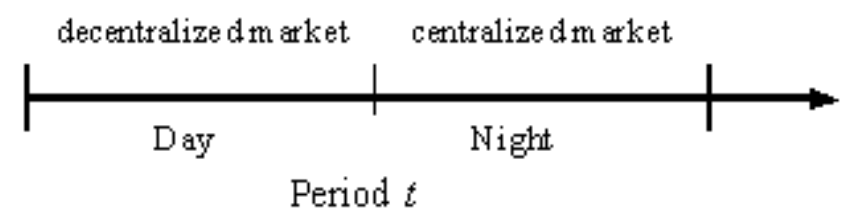

Figure 1: Timing

This completes the description of the physical environment. The timing is illustrated in Figure 1. Given our assumptions, the only feasible trades during the day are barter in special goods and the exchange of special goods for money, and at night the only feasible trades involve general goods and money; special goods can never be traded at night nor general goods during the day because they are only produced in one subperiod and are not storable. ${ }^{5}$ Therefore, in this model money is essential for the same reason it is in the typical search-based model: since meetings in the day market are anonymous there is no scope for trading future promises, so exchange must be quid pro quo. ${ }^{6}$ Notice, however, that from an individual's point of view it is by no means necessary to use money - he could always try to barter special goods directly. It is rather that he may choose to use money because this may make exchange easier.

\footnotetext{
${ }^{5}$ Lagos and Rocheteau (2003) consider an extension where general goods are storable and hence potentially compete with fiat money as a medium of exchange. Although goods are not storable here, we can allow the exchange of intertemporal claims across meetings of the centralized market. Since agents will be homogeneous, as is standard, such claims will not trade but we can still price assets, including real and nominal bonds.

${ }^{6}$ In this context when one says that money is essential it means the economy is able to achieve more outcomes (or better outcomes) with money than without; see Kocherlakota (1998) and Wallace (2001).
} 


\section{Equilibrium}

In this section we build gradually towards the definition of equilibrium. We begin by describing the value functions, taking as given the terms of trade and the distribution of money. In general, the state variable for an individual includes his own money holdings $m$ and a vector of aggregate states $s$. At this point we let $s=(\phi, F)$, where $\phi$ is the value of money in the centralized market $\left(p_{g}=1 / \phi\right.$ is the nominal price at night) and $F$ is the distribution of money holdings in the decentralized market $(F(\tilde{m})$ is the measure of agents in the day market holding $m \leq \tilde{m}$ ). For now the total money stock is fixed at $M$, and so $\int m d F(m)=M$ at every date; we consider changes in the money supply (both deterministic and stochastic) in Section 5. The agent takes as given a law of motion $s_{+1}=\Upsilon(s)$, but it will be determined endogenously in equilibrium. ${ }^{7}$

Let $V(m, s)$ be the value function for an agent with $m$ dollars in the morning when he enters the decentralized market, and $W(m, s)$ the value function in the afternoon when he enters the centralized market. In a singlecoincidence meeting, since the seller's production $h$ must equal the buyer's consumption $x$, we denote their common value by $q(m, \tilde{m}, s)$, and denote the dollars that change hands by $d(m, \tilde{m}, s)$, where in general these may both depend on the money holdings of the buyer $m$ and the seller $\tilde{m}$ as well as the aggregate state $s$. In a double-coincidence meeting, we denote by $B(m, \tilde{m}, s)$ the payoff for an agent with $m$ who meets an agent with $\tilde{m}$. Bellman's

\footnotetext{
${ }^{7}$ Putting $\phi$ in the state vector allows us to capture nonstationary equilibria using recursive methods (Duffie et al. [1994] use a similar approach in overlapping generations models). In any case, for much of this paper we will focus on steady states, so this is not an issue.
} 
equation is ${ }^{8}$

$$
\begin{aligned}
V(m, s)= & \alpha \sigma \int\{u[q(m, \tilde{m}, s)]+W[m-d(m, \tilde{m}, s)]\} d F(\tilde{m}) \\
& +\alpha \sigma \int\{-c[q(\tilde{m}, m, s)]+W[m+d(\tilde{m}, m, s)]\} d F(\tilde{m}) \\
& +\alpha \delta \int B(m, \tilde{m}, s) d F(\tilde{m})+(1-2 \alpha \sigma-\alpha \delta) W(m, s)
\end{aligned}
$$

The value of entering the centralized market with $m$ dollars is

$$
\begin{aligned}
W(m, s) & =\max _{X, H, m_{+1}}\left\{U(X)-A H+\beta V\left(m_{+1}, s_{+1}\right)\right\} \\
\text { s.t. } X & =H+\phi m-\phi m_{+1}
\end{aligned}
$$

where $m_{+1}$ is money taken out of this market. ${ }^{9}$ Normalizing $A=1$ and substituting for $H$,

$$
W(m, s)=\phi m+\max _{X, m_{+1}}\left\{U(X)-X-\phi m_{+1}+\beta V\left(m_{+1}, s_{+1}\right)\right\}
$$

This immediately implies several things. First, $X=X^{*}$ where $U^{\prime}\left(X^{*}\right)=1$. Second, we have the convenient result that $m_{+1}$ does not depend on $m$; intuitively, the quasi-linearity of $\mathcal{U}$ rules out wealth effects in the choice of $m_{+1}$. Third, we have the result that $W$ is linear in $m$,

$$
W(m, s)=W(0, s)+\phi m
$$

Now that we have the value functions, consider the terms of trade in the decentralized market, which are determined by bargaining. There are two

\footnotetext{
${ }^{8}$ The first term is the expected payoff from a single-coincidence meeting where you buy $q(m, \tilde{m}, s)$ and proceed to the centralized market with $m-d(m, \tilde{m}, s)$ dollars. The other terms have similar interpretations.

${ }^{9}$ While standard curvature conditions can be used to guarantee, for example, $X>0$, we cannot so easily rule out corner solutions for $H$ because of quasi-linearity. Our approach is to simply assume an interior solution for $H$ for now, and impose conditions later to guarantee that this is valid.
} 
bargaining situations to consider: single- and double-coincidence meetings. In the latter case we adopt the symmetric Nash bargaining solution with the threat point of an agent given by his continuation value $W(m, s)$. Lemma 1 in the Appendix proves that, regardless of the money holdings of the two agents, in any double-coincidence meeting they give each other the efficient quantity $q^{*}$ defined by $u^{\prime}\left(q^{*}\right)=c^{\prime}\left(q^{*}\right)$, and no money changes hands. Thus, $B(m, \tilde{m}, s)=u\left(q^{*}\right)-c\left(q^{*}\right)+W(m, s)$.

Now consider bargaining in a single-coincidence meeting when the buyer has $m$ and the seller $\tilde{m}$ dollars. Here we use the generalized Nash solution where the buyer has bargaining power $\theta$ and threat points are given by continuation values. ${ }^{10}$ Hence $(q, d)$ maximizes

$$
[u(q)+W(m-d, s)-W(m, s)]^{\theta}[-c(q)+W(\tilde{m}+d, s)-W(\tilde{m}, s)]^{1-\theta}
$$

subject to the feasibility constraint $d \leq m$. By (4) this simplifies nicely to

$$
\max _{q, d}[u(q)-\phi d]^{\theta}[-c(q)+\phi d]^{1-\theta}
$$

subject to $d \leq m$.

Notice (5) does not depend on $\tilde{m}$ at all, and depends on $m$ iff the constraint $d \leq m$ binds. Also, it depends on $s$ only through $\phi$, and indeed only through real balances $z=\phi m$. We write $q(m, \tilde{m}, s)=q(m)$ and $d(m, \tilde{m}, s)=d(m)$ in what follows (the dependence on $\phi$ is implicit). Lemma 2 in the Appendix shows that the solution is

$$
q(m)=\left\{\begin{array}{ll}
\widehat{q}(m) & \text { if } m<m^{*} \\
q^{*} & \text { if } m \geq m^{*}
\end{array} \text { and } d(m)= \begin{cases}m & \text { if } m<m^{*} \\
m^{*} & \text { if } m \geq m^{*}\end{cases}\right.
$$

\footnotetext{
${ }^{10}$ We allow general bargaining power in monetary (single-coincidence) exchange because this will have interesting implications for efficiency and policy; having general bargaining power in barter (double-coincidence) exchange would complicate the presentation without changing anything of substance.
} 


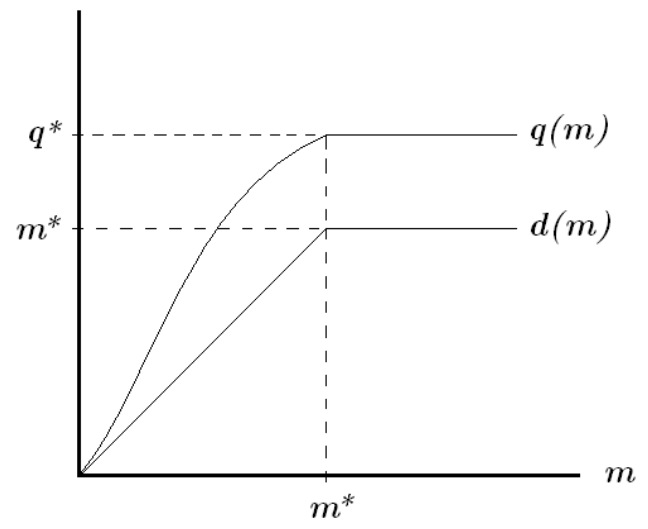

Figure 2: Single-coincidence bargaining solution.

where $\widehat{q}(m)$ solves the first order condition from (5), which for future reference we write as

$$
\phi m=\frac{\theta c(q) u^{\prime}(q)+(1-\theta) u(q) c^{\prime}(q)}{\theta u^{\prime}(q)+(1-\theta) c^{\prime}(q)}=z(q),
$$

and the threshold $m^{*}$ in (6) is given by $m^{*}=z\left(q^{*}\right) / \phi$.

Hence, if real balances are at least $\phi m^{*}$ the buyer gets $q^{*}$; otherwise he spends all his money and gets $\widehat{q}(m)$, which we now show is strictly less than $q^{*}$. Since $u$ and $c$ are $C^{n}$ the implicit function theorem implies that, for all $m<m^{*}, \widehat{q}$ is $C^{n-1}$ and from (7) we have $\widehat{q}^{\prime}=\phi / z^{\prime}(q)$. Inserting $z^{\prime}$ explicitly and simplifying, we find

$$
\widehat{q}^{\prime}=\frac{\phi\left[\theta u^{\prime}+(1-\theta) c^{\prime}\right]^{2}}{u^{\prime} c^{\prime}\left[\theta u^{\prime}+(1-\theta) c^{\prime}\right]+\theta(1-\theta)(u-c)\left(u^{\prime} c^{\prime \prime}-c^{\prime} u^{\prime \prime}\right)} .
$$

Hence, $\widehat{q}^{\prime}>0$ for all $m<m^{*}$. It is easy to check $\lim _{m \rightarrow m^{*}} \widehat{q}(m)=q^{*}$, and so we conclude $\widehat{q}(m)<q^{*}$ for all $m<m^{*}$, as seen in Figure 2 .

We now insert the bargaining outcomes together with $W(m)$ into (1) and 
rewrite Bellman's equation as

$$
V(m, s)=\max _{m_{+1}}\left\{v(m, s)+\phi m-\phi m_{+1}+\beta V\left(m_{+1}, s_{+1}\right)\right\}
$$

where

$$
v(m, s)=v_{0}(s)+\alpha \sigma\{u[q(m)]-\phi d(m)\}
$$

is a bounded and continuous function and $v_{0}(s)$ is independent of $m$ and $m_{+1} \cdot{ }^{11}$ This not only gives us a convenient way to write Bellman's equation, it allows us to establish that there exists a unique $V(m, s)$ in the relevant space of functions satisfying (9), even though this is a nonstandard dynamic programming problem because $V$ is unbounded due to the linear term $\phi m$.

We give the argument here for the case where $s$ is constant - which does nothing to overcome the problem of unboundedness, but does simplify the presentation - and relegate the more general case to Lemma 7 in the Appendix. Given $s$ is constant, write $V(m, s)=\hat{V}(m)$. Then consider the space of functions $\hat{V}: \mathbb{R}_{+} \rightarrow \mathbb{R}$ that can be written $\hat{V}(m)=\hat{v}(m)+\phi m$ for some bounded and continuous function $\hat{v}(m)$. For any two functions in this space $\hat{V}_{1}(m)=\hat{v}_{1}(m)+\phi m$ and $\hat{V}_{2}(m)=\hat{v}_{2}(m)+\phi m$, we can define $\left\|\hat{V}_{1}-\hat{V}_{2}\right\|=\sup _{m \in \mathbb{R}_{+}}\left|\hat{v}_{1}(m)-\hat{v}_{2}(m)\right|$, and this constitutes a complete metric space. One can show the right side of (9) defines a contraction $T \hat{V}$ on the space in question. Hence there exists a unique solution to the functional

\footnotetext{
${ }^{11}$ In any equilibrium $v(m, s)$ is bounded and continuous for the following reason. First, Lemma 5 in the Appendix shows that $F$ is degenerate, and that $\phi_{+1}=\Phi(\phi)$ for some well behaved $\Phi$, in any equilibrium. Lemma 6 shows $\phi$ is bounded. Given this, the bargaining solution implies $v(m, s)$ is bounded and continuous. Although we do not use it below, for the record the term $v_{0}(s)$ is given by

$$
v_{0}(s)=\alpha \sigma \int\{\phi d(\tilde{m})-c[q(\tilde{m})]\} d F(\tilde{m})+\alpha \delta\left[u\left(q^{*}\right)-c\left(q^{*}\right)\right]+U\left(X^{*}\right)-X^{*} .
$$
}


equation $\hat{V}=T \hat{V} .^{12}$

Given that it exists, it is evident from (9) and (10) that $V$ is $C^{n-1}$ with respect to $m$ except at $m=m^{*}$. For $m>m^{*}, V_{m}=\phi$ since $q^{\prime}=d^{\prime}=0$ in this range. For $m<m^{*}$,

$$
V_{m}=\phi+\alpha \sigma\left[u^{\prime}(q) \hat{q}^{\prime}(m)-\phi\right]=(1-\alpha \sigma) \phi+\alpha \sigma u^{\prime}(q) \phi / z^{\prime}(q)
$$

since $\widehat{q}^{\prime}=\phi / z^{\prime}$ and $d^{\prime}=1$ in this range. Inserting $z^{\prime}$ we have

$$
V_{m}=(1-\alpha \sigma) \phi+\frac{\alpha \sigma \phi u^{\prime}\left[\theta u^{\prime}+(1-\theta) c^{\prime}\right]^{2}}{u^{\prime} c^{\prime}\left[\theta u^{\prime}+(1-\theta) c^{\prime}\right]+\theta(1-\theta)(u-c)\left(u^{\prime} c^{\prime \prime}-c^{\prime} u^{\prime \prime}\right)}
$$

for $m<m^{*}$. As $m \rightarrow m^{*}$ from below,

$$
V_{m} \rightarrow(1-\alpha \sigma) \phi+\frac{\alpha \sigma \phi}{1+\theta(1-\theta)(u-c)\left(c^{\prime \prime}-u^{\prime \prime}\right)\left(u^{\prime}\right)^{-2}}<\phi .
$$

Hence, the slope of $V$ with respect to $m$ jumps discretely as we cross $m^{*}$.

The next thing to do is to check the concavity of $V$. At this point we set $c(q)=q$; this reduces notation without affecting the substantive results. Now $V_{m m}$ takes the same sign as $\Gamma+(1-\theta)\left[u^{\prime} u^{\prime \prime \prime}-\left(u^{\prime \prime}\right)^{2}\right]$ for all $m<m^{*}$, where $\Gamma$ is strictly negative but is otherwise of no concern. It is not possible to sign $V_{m m}$ in general, due to the presence of $u^{\prime \prime \prime}$, but this does give us some sufficient conditions for $V_{m m}<0$. One simple condition is $\theta \approx 1$. Another is $u^{\prime} u^{\prime \prime \prime} \leq\left(u^{\prime \prime}\right)^{2}$, which follows if $u^{\prime}$ is log-concave. Hence, we can guarantee that $V$ is strictly concave in $m$ for all $m<m^{*}$, given any $F$ and $\phi{ }^{13}$

\footnotetext{
${ }^{12}$ Operationally, the contraction generates the function $\hat{v}(m)$ and then we simply set $\hat{V}(m)=\hat{v}(m)+\phi m$. Note that this is not the method usually used to deal with unbounded returns, such as that described in Alvarez and Stokey (1998).

${ }^{13}$ To understand the issue, observe that $V_{m m}=\left(q^{\prime}\right)^{2} u^{\prime \prime}+u^{\prime} q^{\prime \prime}$ for all $m<m^{*}$. The first term is negative, but the second takes the sign of $q^{\prime \prime}$, which can be positive. Intuitively, $q^{\prime \prime}>0$ means that having more money gets you a much better deal in bargaining. The assumption $\theta=1$ implies $q(m)=\phi m$ is linear, so $V_{m m}<0$ for sure. If $\theta<1$, however, $q(m)$ is nonlinear and we need a condition like log-concavity to restrict the degree of nonlinearity.
} 
To summarize the analysis so far, we first described the value function in the decentralized market $V(m, s)$ in terms of $W(m, s)$ and the terms of trade. We then derived some properties of the value function in the centralized market $W(m, s)$, including linearity, which made it very easy to solve the bargaining problem for $q(m)$ and $d(m)$. This allowed us to simplify Bellman's equation considerably and establish existence and uniqueness of the solution, and to derive properties of $V$, including differentiability and (under certain assumptions) strict concavity for all $m<m^{*}{ }^{14}$

Given these results we can now solve the problem of an agent deciding how much cash to take out of the centralized market, which from (9) can be summarized as $\max _{m_{+1}}\left\{-\phi m_{+1}+\beta V\left(m_{+1}, s_{+1}\right)\right\}$. First, Lemma 3 in the Appendix proves $\phi \geq \beta \phi_{+1}$ in any equilibrium. This implies $-\phi m_{+1}+$ $\beta V\left(m_{+1}, s_{+1}\right)$ is nonincreasing for $m_{+1}>m_{+1}^{*}$. But recall from (13) that the slope of $V\left(m_{+1}, s_{+1}\right)$ jumps discretely as $m_{+1}$ crosses $m_{+1}^{*}$, as shown in Figure 3. From this it is clear that any solution $m_{+1}$ must be strictly less than $m_{+1}^{*}$. This implies $d=m$ and $q=\widehat{q}(m)<q^{*}$. Moreover, given $V$ is strictly concave for $m_{+1}<m_{+1}^{*}$, there exists a unique maximizer $m_{+1}$. This implies $F$ is degenerate: $m_{+1}=M$ for all agents in any equilibrium. ${ }^{15}$

The first order condition for $m_{+1}$ is

$$
-\phi+\beta V_{1}\left(m_{+1}, s_{+1}\right) \leq 0, \quad=0 \text { if } m_{+1}>0 .
$$

An equilibrium can now be defined as a value function $V(m, s)$ satisfying

\footnotetext{
${ }^{14}$ The existence and uniqueness argument for $V$ was discussed assuming steady state, but the Appendix proves it even if $\phi$ and $F$ vary over time- which is important because it enables us to establish that any equilibrium (not only any stationary equilibrium) has certain features.

${ }^{15}$ Although these results should be clear from the figure, they are proved rigorously in Lemmas 4 and 5 in the Appendix. Note that we do not actually use the value function in these proofs, which allows us to use the results in proving the existence of $V$.
} 


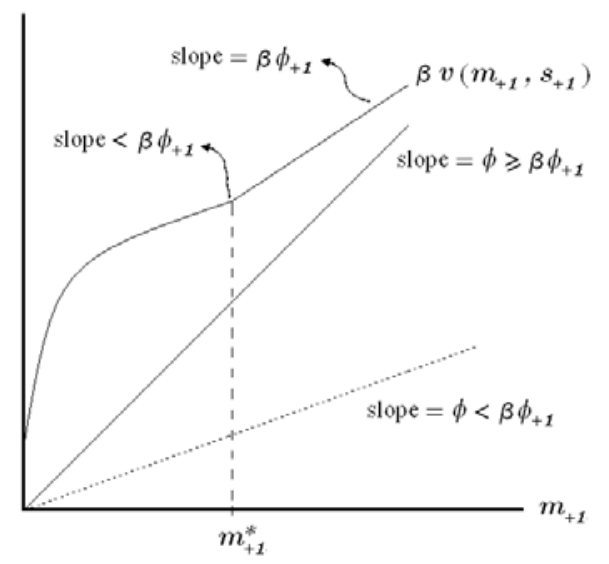

Figure 3: The value function.

Bellman's equation, a solution to the bargaining problem given by $d=m$ and $q=\widehat{q}(m)$, and a bounded path for $\phi$ such that (14) holds at every date with $m=M$. Implicit in this definition is $F$, but it is degenerate. Of course, there is always a nonmonetary equilibrium. In what follows we focus on monetary equilibria, where $\phi>0$ and (14) holds with equality.

We now reduce the equilibrium conditions to one equation in one unknown. First insert $V_{m}$ from (11) into (14) at equality to get

$$
\phi=\beta \phi_{+1}\left[1-\alpha \sigma+\alpha \sigma \frac{u^{\prime}\left(q_{+1}\right)}{z^{\prime}\left(q_{+1}\right)}\right] .
$$

Then insert $\phi=z(q) / M$ from (7) to get

$$
z(q)=\beta z\left(q_{+1}\right)\left[1-\alpha \sigma+\alpha \sigma \frac{u^{\prime}\left(q_{+1}\right)}{z^{\prime}\left(q_{+1}\right)}\right] .
$$

This is a simple difference equation in $q$. A monetary equilibrium can be characterized as any path for $q$ that stays in $\left(0, q^{*}\right)$ and satisfies (15).

Things simplify a lot in some cases. First, consider $\theta=1$ (take-it-orleave-it offers by buyers). In this case, (7) tells us $z(q)=c(q)$, and then (15) 
reduces to ${ }^{16}$

$$
c(q)=\beta c\left(q_{+1}\right)\left[1-\alpha \sigma+\alpha \sigma \frac{u^{\prime}\left(q_{+1}\right)}{c^{\prime}\left(q_{+1}\right)}\right] .
$$

Second, regardless of $\theta$, if we restrict attention to steady states where $q_{+1}=q$, (15) becomes

$$
1=\beta\left[1-\alpha \sigma+\alpha \sigma \frac{u^{\prime}(q)}{z^{\prime}(q)}\right] .
$$

For convenience, we rearrange this as

$$
e(q)=1+\frac{1-\beta}{\alpha \sigma \beta}
$$

where $e(q)=u^{\prime}(q) / z^{\prime}(q)$. From now on we focus on steady states and relegate dynamics to a companion paper, Lagos and Wright (2003).

Consider first steady states with $\theta=1$, which means $z(q)=c(q)$ and therefore (16) becomes

$$
\frac{u^{\prime}(q)}{c^{\prime}(q)}=1+\frac{1-\beta}{\alpha \sigma \beta}
$$

Since $\frac{u^{\prime}\left(q^{*}\right)}{c^{\prime}\left(q^{*}\right)}=1<1+\frac{1-\beta}{\alpha \sigma \beta}$, a monetary steady state $q^{s} \in\left(0, q^{*}\right)$ exists iff $\frac{u^{\prime}(0)}{c^{\prime}(0)}>1+\frac{1-\beta}{\alpha \sigma \beta}$, and if it exists it is obviously unique. More generally, for any $\theta$ a monetary steady state exists if $e(0)>1+\frac{1-\beta}{\alpha \sigma \beta}$, but we cannot be sure of uniqueness since we do not know the sign of $e^{\prime}$. However, one can show that $u^{\prime} \log$-concave or $\theta \approx 1$ implies $e^{\prime}<0$ and uniqueness. One can also show $e(q)$ is increasing in $\theta$; hence if $q^{s}$ is unique then $\partial q^{s} / \partial \theta>0$. It is also clear that $\partial q^{s} / \partial \alpha>0, \partial q^{s} / \partial \sigma$ and $\partial q^{s} / \partial \beta>0$. For $\theta=1$, notice $q^{s} \rightarrow q^{*}$ as $\beta \rightarrow 1$; for $\theta<1$, however, $q$ is bounded away from $q^{*}$ even in the limit as $\beta \rightarrow 1$.

We summarize the main findings in a Proposition. The proof follows from the discussion in the text, although two technical claims in the previous

\footnotetext{
${ }^{16}$ We assumed above that $c(q)=q$ but we write the formula for a general function $c(q)$ to emphasize the symmetry.
} 
paragraph need to be established: $e$ is increasing in $\theta$, and $e$ is decreasing in $q$ if $u^{\prime}$ is log-concave or $\theta \approx 1$. This is done in the Appendix.

Proposition 1 Any monetary equilibrium implies that $\forall t>0, m=M$ with probability 1 ( $F$ degenerate), $d=m$, and $q=\widehat{q}(m)<q^{*}$. Given any $\theta>0$, a steady state $q^{s}>0$ exists if $e(0)>1+\frac{1-\beta}{\alpha \sigma \beta}$. It is unique if $\theta \approx 1$ or $u^{\prime}$ is log-concave, in which case $q^{s}$ is increasing in $\beta, \alpha, \sigma$ and $\theta$. It converges to $q^{*}$ as $\beta \rightarrow 1$ iff $\theta=1$.

Before proceeding, recall that so far we have simply assumed an interior solution for $H$; we now give conditions to guarantee that this is valid. Suppose first that we want to be sure $H>0$. Assume the economy begins at $t=0$ with the centralized market, and let $\bar{m}_{0}$ be the upper bound of the support of the (exogenous) initial distribution on money, $F_{0}$. When we ignored nonnegativity we found $X=X^{*}$ and $m_{+1}=M$, so an agent endowed with $m$ supplies $H(m)=X^{*}+\phi(M-m)$ hours. From $(7), \phi$ is an increasing function of $q$ in equilibrium, and since $q<q^{*}$, $\phi$ is bounded above by $\phi^{*}=z\left(q^{*}\right) / M$. Hence, in the worst case scenario $\phi=\phi^{*}$, we have $H(m)>0$ for all $m$ in the support of $F_{0}$ if

$$
\bar{m}_{0}<M+\frac{X^{*}}{\phi^{*}}=M\left[1+\frac{X^{*}}{z\left(q^{*}\right)}\right] .
$$

One can regard (18) as a restriction on the exogenous $F_{0}$ (it cannot be too disperse). For $t \geq 1$, the distribution $F_{t}$ is endogenous with an upper bound of $\bar{m}_{t}=2 M$ in equilibrium. Hence, to guarantee $H_{t}>0$ we need (18) to hold at $\bar{m}_{t}=2 M$, or which reduces to $X^{*}>z\left(q^{*}\right)$. Similarly one can show $H_{t}<\bar{H}$ for all $t \geq 0$, where $\bar{H}$ is an upper bound on labor supply, if $X^{*}+z\left(q^{*}\right)<\bar{H}$. Hence we have simple conditions to guarantee $0<H_{t}<\bar{H}$ for all $t$. Of course, it could be interesting to consider cases where we do not have interior 
solutions, and therefore $F$ is not degenerate, just as it would be interesting to consider preferences that are not quasi-linear or centralized markets that do not convene every period; the goal here, however, is to develop a model that is analytically tractable precisely because $F$ is degenerate in equilibrium.

Next, we emphasize that so far we have dealt only with deterministic environments. We now show that with stochastic shocks the constraint $d \leq$ $m$ does not have to bind with probability 1 . Consider first match-specific uncertainty: when two agents meet in a single coincidence, they draw $\varepsilon=$ $\left(\varepsilon_{b}, \varepsilon_{s}\right)$ from $H(\varepsilon)$, independently across meetings; in that meeting utility from consumption will be $\varepsilon_{b} u(q)$ and disutility from production will be $\varepsilon_{s} q$. For simplicity, we assume $\theta=1$ (take-it-or-leave-it offers), $\delta=0$ (no barter), and $u^{\prime}(0)=\infty$. Then (6) becomes

$$
q(m)=\left\{\begin{array}{ll}
\widehat{q}(m, \varepsilon) & \text { if } m<m^{*}(\varepsilon) \\
q^{*}(\varepsilon) & \text { if } m \geq m^{*}(\varepsilon)
\end{array} \text { and } d(m)= \begin{cases}m & \text { if } m<m^{*}(\varepsilon) \\
m^{*}(\varepsilon) & \text { if } m \geq m^{*}(\varepsilon)\end{cases}\right.
$$

where $\widehat{q}(m, \varepsilon)=\phi m / \varepsilon_{s}, u^{\prime}\left[q^{*}(\varepsilon)\right]=\varepsilon_{s} / \varepsilon_{b}$, and $m^{*}(\varepsilon)=\varepsilon_{s} q^{*}(\varepsilon) / \phi$.

For a given $\varepsilon_{s}$, buyers with high realizations of $\varepsilon_{b}$ will spend all their cash but those with low $\varepsilon_{b}$ may not. Let $C=\left\{\varepsilon \mid q^{*}(\varepsilon)>\phi m / \varepsilon_{s}\right\}$ be the set of realizations such that $d=m$. Bellman's equation is still given by (9) but now

$$
v(m, s)=\alpha \sigma \int\left\{\varepsilon_{b} u[q(m, \varepsilon)]-\phi d(m, \varepsilon)\right\} d H(\varepsilon)+U\left(X^{*}\right)-X^{*} .
$$

Since $v_{m m}<0, V$ is strictly concave and again $F$ is degenerate. Substituting $V_{m}$ into the first order condition $\phi=\beta V_{m}$, we get

$$
\int_{C}\left[\left(\frac{\varepsilon_{b}}{\varepsilon_{s}}\right) u^{\prime}\left(\frac{M \phi_{+1}}{\varepsilon_{s}}\right)-1\right] d H(\varepsilon)=\frac{\phi-\beta \phi_{+1}}{\alpha \sigma \beta \phi_{+1}} .
$$

There exists a unique monetary steady state $\phi^{s}>0$, and from this $q=$ $q(M, \varepsilon)$ and $d=d(M, \varepsilon)$ are obtained from the bargaining solution. Clearly 
$d \leq m$ must bind with positive probability, since otherwise (19) could not hold. However, it is easy to work out examples where it binds with probability less than $1 .{ }^{17}$

We can also allow permanent differences across agents. Suppose, for example, that type $\varepsilon$ agents have utility $\varepsilon u(q)$, and there is an exogenous distribution of types $G(\varepsilon)$. In other ways agents are homogeneous, and the economy is deterministic. For simplicity we again set $\theta=1, c(q)=q$ and $\delta=0$. Each type $\varepsilon$ solves a problem leading in steady state to a version of (17) given by $u^{\prime}\left(q_{\varepsilon}\right)=1+\frac{1-\beta}{\alpha \sigma \beta}$, where from the bargaining solution with $\theta=1, q_{\varepsilon}=\phi m_{\varepsilon}$. That is, type $\varepsilon$ will have "money demand"

$$
\phi m_{\varepsilon}=u^{\prime-1}\left(\frac{\alpha \sigma \beta+1-\beta}{\alpha \sigma \beta \varepsilon}\right) .
$$

Agents with higher $\varepsilon$ will hold more money, but all agents of the same type will hold the same $m_{\varepsilon}$ in equilibrium. The aggregate distribution $F$ is no longer degenerate, but is fully determined by the exogenous type distribution $G$, which is relatively simple compared to some models to be discussed in the next section.

\footnotetext{
${ }^{17}$ Rather than interpreting $\varepsilon$ as match-specific, suppose it is an aggregate shock with conditional distribution $H\left(\varepsilon_{+1} \mid \varepsilon\right)$. Bellman's equation then satisfies a version of $(9)$ where

$$
v(m, \varepsilon)=\alpha \sigma\left\{\varepsilon_{b} u[q(m, \varepsilon)]-\phi d(m, \varepsilon)\right\}+U\left(X^{*}\right)-X^{*} .
$$

Substituting $V_{m}$ into $\phi=\beta V_{m}$, we get

$$
\phi(\varepsilon)=\beta \int \phi\left(\varepsilon_{+1}\right)\left(1+I\left(\varepsilon_{+1}\right) \alpha \sigma\left\{\frac{\varepsilon_{+1, b}}{\varepsilon_{+1, s}} u^{\prime}\left[\frac{\phi\left(\varepsilon_{+1}\right) M}{\varepsilon_{+1, s}}\right]-1\right\}\right) d H\left(\varepsilon_{+1} \mid \varepsilon\right)
$$

where $I(\varepsilon)$ is an indicator function that equals 1 if $\varepsilon \in C$ and 0 otherwise. In general this is a functional equation in $\phi(\cdot)$. In the i.i.d. case the right side is independent of $\varepsilon$, so $\phi(\varepsilon)$ is constant and things are exactly the same as the match-specific case.
} 


\section{Discussion}

Trejos and Wright (1995) describe a version of their model where $m \in \mathbb{R}_{+}$, and present a Bellman equation similar to (1), except that since there are no centralized markets, $W(m)=\beta V(m)$. With no centralized markets the distribution $F$ is not degenerate. Trejos and Wright (1995) cannot solve that model, and only analyze the case $m \in\{0,1\}$ - obviously a severe restriction. Molico (1999) studies the model with $m \in \mathbb{R}_{+}$numerically. ${ }^{18}$ Although his computational results are very interesting, there is something to be said for analytic tractability. For one thing, if one has to resort to computation it is difficult to say much about existence, uniqueness or multiplicity, dynamics, and many other issues of interest in monetary economics. The analysis here is much simpler due to the presence of centralized markets. This assumption makes $W$ linear, makes bargaining easy, and makes $F$ degenerate.

It is important to mention at this point that there is an alternative approach due to Shi (1997) that also yields a degenerate $F$, but for a different reason. His model assumes the fundamental decision-making unit is not an individual, but a family with a continuum of agents. Each household's members search in decentralized markets, but at the end of each round of search they meet back at the homestead where they can share their money. By the law of large numbers each family has the same total amount of money, and in equilibrium the family divides it evenly among its buyers for the next round. Hence, all buyers in the decentralized market have the same $m$. The largehousehold "trick" accomplishes the same result as our centralized market - it

\footnotetext{
${ }^{18}$ Other papers that relax the restriction $m \in\{0,1\}$ and try to deal with a nondegenerate $F$ include Green and Zhou (1998), Zhou (1999), Camera and Corbae (1999), Taber and Wallace (1999), and Berentsen (2002).
} 
renders $F$ degenerate. ${ }^{19}$

While both approaches may be useful, it seems incumbent upon us to discuss the relative merits of our "trick" as compared to Shi's. First, some people seem to view the infinite family structure as unappealing for a variety of reasons. Whether or not one agrees with this point of view, it seems good to have an alternative lest people think that tractable monetary models with search-theoretic foundations require infinite families. They do not. Second, there are some serious technical complications that arise in family models because infinitesimal agents bargain over trades that benefit larger decisionmaking units. Rather than get into the details we refer the reader to Rauch (2000) and Berentsen and Rocheteau (2002). These technical problems with bargaining do not arise here since individuals bargain for themselves; thus we can use standard theory, and indeed the linearity of $W$ makes bargaining extremely simple.

Third, there is the related but distinct point that individual incentive conditions are not taken into account in family models: agents act not in their own self interest, but in accordance with rules prescribed by the head of the household. Every time an individual produces he suffers a cost. If he reported back to the clan without the cash and claimed he had no customers, this would save the cost with no implication for his future payoff. For the family structure to survive, then, agents must act in the interest of the household. Although this is not necessarily a fatal flaw, it is worth emphasizing because some people misinterpret Shi's structure as equivalent to competitive insurance markets. It is not. With competitive markets the

\footnotetext{
${ }^{19}$ Applications of this approach are contained in Shi $(1998,1999)$, Rauch (2000), Berentsen, Rocheteau and Shi (2001), Faig (2001), and Head and Kumar (2005).
} 
moral hazard problem precludes insurance. The moral hazard problem also precludes insurance here, but because agents adjust $m$ through spot trades in the centralized market we get $F$ degenerate without insurance.

Fourth, we simply find our model more transparent and easier to use. It is widely thought that a problem with search-based macro theory is that it is too far removed from "standard" models. A relatively sympathetic version of this view is expressed in Kiyotaki and Moore (2001): "The matching models are without doubt ingenious and beautiful. But it is quite hard to integrate them with the rest of macroeconomic theory - not least because they jettison the basic tool of our trade, competitive markets." We think it is a virtue of our approach that competitive markets are being brought back on board. In particular, for many extensions and applications one may want to introduce market trading anyway (e.g., bond, capital, or labor markets). In our model the markets are already up and running, so no additional structure needs to be imposed, and large families would actually be redundant.

Having said all this, we reiterate that the family model and centralized markets are both potentially useful devices. Moreover, we acknowledge that in addition to these markets we also need special preferences: given more general utility, agents will still adjust $m$ in the centralized market, but only with quasi-linearity will they necessarily all adjust to $m=M \cdot{ }^{20}$ Although our

${ }^{20}$ If we replace $U(X)-A H$ with $\hat{U}(X, \bar{H}-H)$, it is an exercise to show

$$
\frac{\partial H}{\partial m}=\frac{-\beta V^{\prime \prime}}{\Delta}\left(w \hat{U}_{11}-\hat{U}_{12}\right), \frac{\partial m_{+1}}{\partial m}=\frac{\phi}{\Delta}\left(\hat{U}_{11} \hat{U}_{22}-\hat{U}_{12}^{2}\right), \frac{\partial X}{\partial m}=\frac{\beta V^{\prime \prime}}{\Delta}\left(\hat{U}_{22}-w \hat{U}_{12}\right),
$$

where $w$ is the wage and $\Delta>0$. Hence, for example, $\partial H / \partial m<0$ iff leisure is normal in the usual sense. Notice $\partial m_{+1} / \partial m \geq 0$ regardless of $\hat{U}$, and $\partial m_{+1} / \partial m=0$ iff either $H$ or $X$ enters $\hat{U}$ linearly. If $H$ enters linearly, when we give the agent more $m, X$ stays the same and $H$ adjusts to satisfy the budget constraint, while if $X$ enters linearly the opposite happens. 
preferences are special they are not bizarre. Models where utility is linear in leisure have been used in many well-known macro and monetary applications, including Hansen (1985), Rogerson (1988), Cooley and Hansen (1989, 1991), and Christiano and Eichenbaum (1992). What is critical here is that we can ignore wealth effects in the sense that the amount of money you take to the market today does not depend on how much you spent yesterday. This does not seem empirically implausible. Moreover, as long as wealth effects are not too big the substantive predictions of our model will be approximately correct, even though the gain in analytic tractability accrues only in the limit when wealth effects are zero. ${ }^{21}$

\section{Changes in Money Supply}

We begin this section by generalizing the model to allow the money supply to grow over time, say $M_{+1}=(1+\tau) M$. New money is injected as a lumpsum transfer, or tax if $\tau<0$, that occurs after agents leave the centralized market. Bellman's equation becomes

$$
V(m, \phi)=\max _{m_{+1}}\left\{v(m, \phi)+\phi m-\phi m_{+1}+\beta V\left(m_{+1}+\tau M, \phi_{+1}\right)\right\}
$$

where $v$ is defined in (10), and we write $s=\phi$ since $F$ will again be degenerate. In general $\tau$ could vary with time, but if it is constant then it makes sense to consider steady states where $q$ and real balances $z=\phi M$ are

\footnotetext{
${ }^{21}$ Of course continuity needs to be established. Imagine the model where we replace $U(X)-A H$ by $U(X)-A H^{\chi}$ but everything else is the same. For $\chi>1, F$ is not degenerate in equilibrium, and we need to use numerical methods. The computational experiments in Khan, Thomas and Wright (2003) show that for $\chi$ not too far from 1 the numerical results for the general case are close to the analytic results one can derive for the case $\chi=1$.
} 
constant; that is, $\phi_{+1}=\phi /(1+\tau)$. As in the previous section, $\phi \geq \beta \phi_{+1}$ is necessary for an equilibrium to exist (Lemma 3), and so $\tau \geq \beta-1$.

Following the same procedure as before, we insert $V_{m}$ and $\phi=z(q) / M$ into $\phi=\beta V_{m}$ to get the generalized version of (15):

$$
\frac{z(q)}{M}=\beta \frac{z\left(q_{+1}\right)}{M_{+1}}\left[1-\alpha \sigma+\alpha \sigma \frac{u^{\prime}\left(q_{+1}\right)}{z^{\prime}\left(q_{+1}\right)}\right] .
$$

Given $M_{+1}=(1+\tau) M$ with $\tau$ constant, if we focus on steady states then after some algebra we get the generalized version of (16)

$$
e(q)=1+\frac{1-\beta+\tau}{\alpha \sigma \beta},
$$

where again $e(q)=u^{\prime}(q) / z^{\prime}(q)$. Assuming a unique monetary steady state $q^{s}$ exists, which it will under the conditions given in Proposition 1, we have $\partial q^{s} / \partial \tau<0$.

If $\theta=1$ then $z(q)=c(q)$ and hence the efficient solution $q^{s}=q^{*}$ obtains iff $\tau=\tau^{F}=\beta-1$. This is the Friedman Rule: deflate at the rate of time preference. ${ }^{22}$ If $\theta<1$, however, then $q^{s}<q^{*}$ at the Friedman Rule. Since $\tau \geq \tau^{F}$ is a necessary condition for equilibrium to exist, and $\partial q^{s} / \partial \tau<0$, the Friedman Rule is still optimal but it does not achieve the first best $q^{*}$ unless $\theta=1$. The reason is that in this model there are two types of inefficiencies, one due to $\beta$ and one to $\theta$. The $\beta$ effect is standard: when you acquire cash you get a claim to future consumption, but because $\beta<1$ you are willing to produce less for cash than the $q^{*}$ you would produce if you could turn the proceeds into immediate consumption. The Friedman Rule corrects this by generating a real return on money that compensates for discounting. ${ }^{23}$

\footnotetext{
${ }^{22}$ One can also write $(22)$ as $e(q)=1+i / \alpha \sigma \beta$, where $i$ is the nominal interest rate (see Section 6), and the Friedman Rule can equivalently be stated as $i=0$.

${ }^{23}$ Although the $\beta$ effect is somewhat standard, our model does say some novel things about it because the frictions show up explicitly; e.g., (22) makes it clear that for a given $\beta$ and $\tau$ the inefficiency gets worse as $\alpha \sigma$ gets smaller.
} 


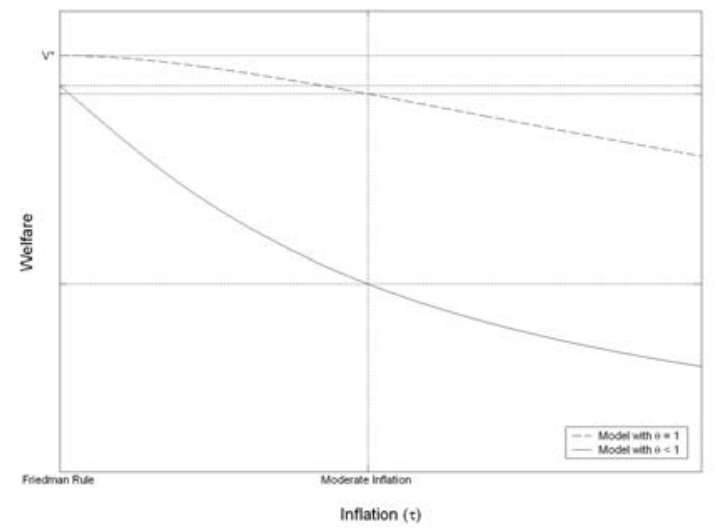

Figure 4: The welfare cost of inflation.

The more novel effect here is the wedge due to $\theta<1$. One intuition for this is the notion of a holdup problem. An agent who carries a dollar into next period is making an investment with cost $\phi$ (he could have spent the cash on general goods). When he uses the money in the future he reaps the full return on his investment iff $\theta=1$; otherwise the seller "steals" part of the surplus. Thus, $\theta<1$ reduces the incentive to invest, which lowers the demand for money and hence $q$. Therefore $\theta<1$ implies $q^{s}<q^{*}$ even at the Friedman Rule. The Hosios' (1990) condition for efficiency says the bargaining solution should split the surplus so that each party is compensated for their contribution to the surplus in the match. Here the surplus in a singlecoincidence meeting is all due to the buyer, since the outcome depends on $m$ but not $\tilde{m}$. Hence, efficiency requires $\theta=1$.

The wedge due to $\theta<1$ can be very important for issues like the welfare cost of inflation. We can measure welfare by the payoff of the representative agent $V$. When $\theta=1, V$ is maximized at the Friedman Rule $\tau^{F}$ and it 
achieves the efficient outcome:

$$
(1-\beta) V^{*}=\alpha(\delta+\sigma)\left[u\left(q^{*}\right)-c\left(q^{*}\right)\right]+U\left(X^{*}\right)-X^{*}
$$

See Figure 4. With $\theta=1$, small deviations from $\tau^{F}$ have very small effects on $V$ due to the Envelope Theorem, exactly as in the typical reduced-form (e.g., cash-in-advance) model. When $\theta<1, \tau^{F}$ is still optimal but it is a constrained optimum: $\tau<\tau^{F}$ would achieve a higher $q$ and $V$ if it were feasible, but it is not. Hence the slope of $V$ with respect to $\tau$ is steep at $\tau^{F}$ and the envelope theorem does not apply. A moderate inflation therefore has a bigger welfare cost when $\theta<1$. In Section 6 we quantify this.

Before moving to numerical exercises we want to discuss the effects of uncertainty in the $M$. Consider first random transfers across agents: before the start of trade, an agent who brought $m$ to the decentralized market ends up with $m+\rho$ dollars, where $\rho$ has distribution $H(\rho)$ on $[\underline{\rho}, \bar{\rho}]$ with $E \rho=0$. Here $E \rho=0$ is assumed to disentangle redistribution from average money growth effects. Bellman's equation is still given by (9) but now

$$
v(m, s)=U\left(X^{*}\right)-X^{*}+\alpha \sigma \int_{\underline{\rho}}^{\bar{\rho}}\{u[q(m+\rho)]-\phi d(m+\rho)\} d H(\rho) .
$$

Hence, $v_{m m}<0$ and $F$ is degenerate. We again assume $\theta=1$ and $u^{\prime}(0)=\infty$ here. Substituting $V_{m}$ into $\phi=\beta V_{m}$ yields

$$
\int_{\underline{\rho}}^{\hat{\rho}}\left\{u^{\prime}\left[(M+\rho) \phi_{+1}\right]-1\right\} d H(\rho)=\frac{\phi-\beta \phi_{+1}}{\alpha \sigma \beta \phi_{+1}}
$$

where $\hat{\rho}=q^{*} / \phi_{+1}-M$ is the minimum transfer that makes $d \leq m$ slack. There exists a unique monetary steady state $\phi^{s}$.

Now consider a family of distributions $H(\rho, \Sigma)$ where increasing $\Sigma$ implies a mean preserving spread in $H$. With some work, one can show $\partial \phi^{s} / \partial \Sigma$ is 
equal in sign to

$$
-\phi u^{\prime \prime}\left(q^{*}\right) \Xi(\hat{\rho}, \Sigma)+\int_{\underline{\rho}}^{\hat{\rho}} \phi^{2} u^{\prime \prime \prime}[(M+\rho) \phi] \Xi(\rho, \Sigma) d \rho,
$$

where $\Xi(\tilde{\rho}, \Sigma)=\int_{\underline{\rho}}^{\widetilde{\rho}} H_{2}(\rho, \Sigma) d \rho \geq 0$. The first term is positive but the second depends on $u^{\prime \prime \prime}$. As long as $u^{\prime \prime \prime} \geq 0$ more risk increases $\phi^{s}$ and hence $q^{s}$ due to what may be called the "precautionary demand for money" but one can verify this unambiguously reduces welfare. This contrasts with models where the distribution $F$ is nondegenerate and random transfers may be welfare improving (Molico [1999]; Berentsen [2002]). The reason is that in those models random transfers can make $F$ less unequal; this effect obviously cannot occur when $F$ is degenerate.

Another experiment is to let the growth rate of $M$ be random with distribution $H\left(\tau_{+1} \mid \tau\right)$. Bellman's equation now satisfies (9) with

$$
v(m, \tau)=U\left(X^{*}\right)-X^{*}+\alpha \sigma\{u[q(m)]-\phi d(m)\} .
$$

Again $F$ is degenerate, and the usual procedure yields

$$
\phi=\beta \int_{C^{c}} \phi_{+1} d H\left(\tau_{+1} \mid \tau\right)+\beta \int_{C} \phi_{+1}\left[\alpha \sigma u^{\prime}\left(\phi_{+1} m_{+1}\right)+1-\alpha \sigma\right] d H\left(\tau_{+1} \mid \tau\right)
$$

where $C=\left\{\tau \mid(m+\tau M) \phi<q^{*}\right\}$. If we focus on stationary equilibrium,

$$
z(\tau)=\beta \int_{C^{c}} \frac{z\left(\tau_{+1}\right) d H\left(\tau_{+1} \mid \tau\right)}{1+\tau_{+1}}+\beta \int_{C} \frac{\left\{\alpha \sigma u^{\prime}\left[z\left(\tau_{+1}\right)\right]+1-\alpha \sigma\right\} z\left(\tau_{+1}\right) d H\left(\tau_{+1} \mid \tau\right)}{1+\tau_{+1}}
$$

where $z(\tau)$ is real balances in state $\tau$. This is a functional equation in $z(\cdot)$.

If shocks are i.i.d., $z(\tau)$ is constant and the constraint binds with probability 1 ; in this case $z$ solves

$$
u^{\prime}(z)=1+\frac{\zeta^{-1}-\beta}{\beta \alpha \sigma}
$$




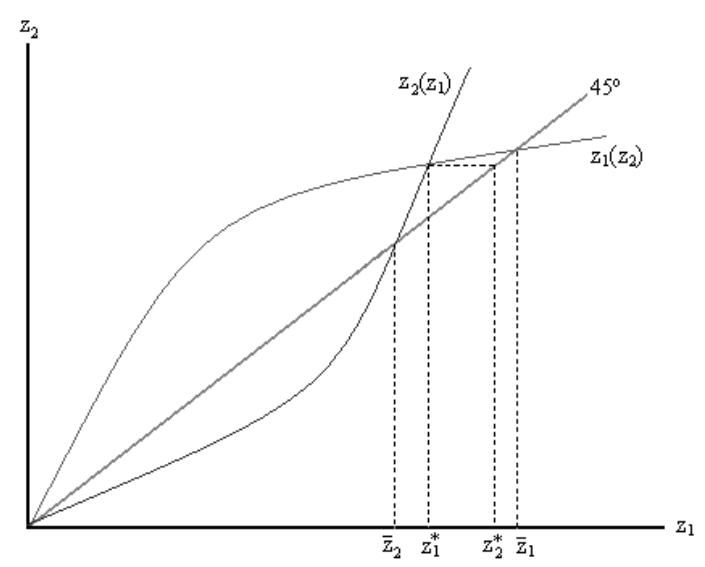

Figure 5: Equilibrium with random $\tau$.

where $\zeta=\int(1+\tau)^{-1} d H(\tau)$. When $\tau$ is persistent the inflation forecast depends on $\tau$ and so does $z$. Suppose, for example, $\tau \in\left\{\tau_{1}, \tau_{2}\right\}$ with $\tau_{1}>\tau_{2}$, $\operatorname{pr}\left(\tau=\tau_{i} \mid \tau_{i}\right)=p_{i}, \operatorname{pr}\left(\tau=\tau_{1} \mid \tau_{2}\right)=s_{2}$ and $\operatorname{pr}\left(\tau=\tau_{2} \mid \tau_{1}\right)=s_{1}$ where $p_{1}>s_{2}$ (persistence). We write $(24)$ as two equations in $\left(z_{1}, z_{2}\right)$, and look for a solution $\left(z_{1}^{*}, z_{2}^{*}\right)$ such that $z_{i}^{*}<q^{*}$. These equations, shown in Figure 5, can be rearranged as

$$
\begin{aligned}
& z_{1}=z_{1}\left(z_{2}\right)=\left[\frac{p_{1}}{s_{2}}-\frac{\beta(1-\alpha \sigma)\left(p_{1} p_{2}-s_{1} s_{2}\right)}{s_{2}\left(1+\tau_{2}\right)}\right] z_{2}-\frac{\beta \alpha \sigma\left(p_{1} p_{2}-s_{1} s_{2}\right)}{s_{2}\left(1+\tau_{2}\right)} u^{\prime}\left(z_{2}\right) z_{2} \\
& z_{2}=z_{2}\left(z_{1}\right)=\left[\frac{p_{2}}{s_{1}}-\frac{\beta(1-\alpha \sigma)\left(p_{1} p_{2}-s_{1} s_{2}\right)}{s_{1}\left(1+\tau_{1}\right)}\right] z_{1}-\frac{\beta \alpha \sigma\left(p_{1} p_{2}-s_{1} s_{2}\right)}{s_{1}\left(1+\tau_{1}\right)} u^{\prime}\left(z_{1}\right) z_{1} .
\end{aligned}
$$

Notice $z_{i}(0)=0$ and $\lim _{z \rightarrow \infty} z_{i}(z)=\infty$. It may be shown that $z_{i}^{\prime}>0$ as long as $-u^{\prime \prime}(z) z / u^{\prime}(z) \geq 1$. Let $\bar{z}_{i}=z_{i}\left(\bar{z}_{i}\right)$ be the point where the $z_{i}(\cdot)$ function crosses the $45^{\circ}$ line, as seen in Figure 5 , given by the solutions to

$$
\begin{aligned}
& 1+\alpha \sigma\left[u^{\prime}\left(\bar{z}_{2}\right)-1\right]=\frac{\left(1+\tau_{1}\right)\left(p_{2}-s_{1}\right)}{\beta\left(p_{1} p_{2}-s_{1} s_{2}\right)} \\
& 1+\alpha \sigma\left[u^{\prime}\left(\bar{z}_{1}\right)-1\right]=\frac{\left(1+\tau_{2}\right)\left(p_{2}-s_{1}\right)}{\beta\left(p_{1} p_{2}-s_{1} s_{2}\right)} .
\end{aligned}
$$


These imply $\bar{z}_{2}<\bar{z}_{1}$ if $\tau_{1}>\tau_{2}$, and given $z_{i}^{\prime}>0$ this implies $z_{1}^{*}<z_{2}^{*}$. Hence, when the shocks to the money supply are persistent, real balances are smaller in periods of high inflation, simply because in these periods beliefs about future inflation are higher. ${ }^{24}$ It may be interesting to purse these models with uncertainty, but in what follows we return to deterministic models and consider the welfare cost of perfectly predictable inflation.

\section{Quantitative Analysis}

The preference structure used above is $\mathcal{U}(x, h, X, H)=u(x)-c(h)+U(X)-$ $A H$. We specialize things for the quantitative work as follows. First,

$$
u(q)=\frac{(q+b)^{1-\eta}-b^{1-\eta}}{1-\eta}
$$

where $\eta>0$ and $b \in(0,1)$. This generalizes the standard constant relative risk aversion preferences by including the parameter $b$, which forces $u(0)=0 .{ }^{25}$ We will actually set $b \approx 0$ below to make this close to the usual preferences; hence $q^{*}=1-b \approx 1$. Next, $U(X)=B \log (X)$; therefore preferences over centralized market goods here are exactly the same as Cooley and Hansen (1989), $B \log (X)-A H$. Finally, to make the disutility of labor the same in the two markets we set $c(h)=A h$, and normalize $A=1$ (we could calibrate $A$ to match total hours worked in the data but this affects none of the welfare calculations). Notice $A=1$ implies $X^{*}=B$.

\footnotetext{
${ }^{24}$ One detail remains: recall that the equilibrium was constructed conjecturing $z_{i}^{*}<q^{*}$. Since $z_{i}^{*}<\bar{z}_{1}$, for the conjecture to be correct it is sufficient to ensure $\bar{z}_{1} \leq q^{*}$, which holds iff $\left(1+\tau_{2}\right)\left(p_{1}-s_{2}\right) \geq \beta\left(p_{1} p_{2}-s_{1} s_{2}\right)$.

${ }^{25}$ We require $u(0)=0$ because we want the utility of consuming $q=0$ to be the same as the utility of not consuming; this is relevant here because each period agents get to consume day goods with probability less than 1 . We assume $b \in(0,1)$ to guarantee $u^{\prime}(q)>0$ and $q^{*}>0$.
} 
The period length can basically be anything, but we begin with a year mainly to facilitate comparison with Lucas (2000); below we show a monthly model yields very similar results. Hence, we set $r=0.04$ for now. In terms of arrival rates, we can normalize $\alpha=1$ since it is only the products $\alpha \delta$ and $\alpha \sigma$ that matter. We set $\delta=0$ since barter is relatively rare (this does not matter for the results). For $\sigma$, one might think it can be calibrated to match velocity $\nu$, but theory does not pin down how much cash is used in the centralized market. ${ }^{26}$ Hence, we again follow Lucas and set $\sigma$ as well as the preference parameters to match the "money demand" data - that is, the relationship between the nominal interest rate $i$ and $L=M / P Y=1 / \nu$. This relationship represents "money demand" in the sense that desired real balances $M / P$ are proportional to $Y$, with a factor of proportionality $L$ that depends on the cost of holding cash $i$.

In the model, nominal output in the centralized market is $X^{*} / \phi=B / \phi$ and nominal output in the decentralized market is $\sigma M$. Hence $P Y=B / \phi+$ $\sigma M$ and $Y=B+\sigma \phi M$. In equilibrium, $Y=B+\sigma z(q)$ and

$$
L=\frac{M / P}{Y}=\frac{z(q)}{B+\sigma z(q)} .
$$

Moreover, in steady state

$$
\frac{u^{\prime}(q)}{z^{\prime}(q)}=1+\frac{1-\beta+\tau}{\sigma \beta}=1+\frac{i}{\sigma},
$$

and after performing the usual substitutions, $\beta=1 /(1+r)$ and $(1+r)(1+\tau)=$

\footnotetext{
${ }^{26}$ Given their budget constraints, agents are indifferent between receiving wages in terms of general goods or dollars in the night market, since both can be used within the subperiod. If one assumes dollars are used at night only for trades to adjust $m$ velocity is $\nu=2 \sigma$ (because each dollar turns over once in every single-coincidence meeting during the day and once more at night). At the opposite extreme, if one assumes cash is used in every transaction in the centralized market, then $\nu=P Y / M$, where $Y$ is real output aggregated across markets and $P$ the nominal price level.
} 
$1+i$. This implies $q=q(i)$, and therefore

$$
L=\frac{z[q(i)]}{B+\sigma z[q(i)]}=L(i) .
$$

This is our "money demand" curve: $L(i)=M / P Y$.

Our approach is to try to fit (25) to annual observations by choosing $\Omega=(\eta, B, \sigma)$, where in the data $i$ is the commercial paper rate and $L$ is constructed using $G D P$, the $G D P$ deflator, and $M 1$, exactly as in Lucas. The sample period is $1900-2000$. We do not include $\theta$ in $\Omega$; rather we will calibrate to alternative values of $\theta$, as discussed below. The result when $\theta=1$ is shown in Figure 6, which generates a very reasonable fit with $\Omega=$ $(0.266,2.133,0.311)$. However, $\Omega$ is not precisely identified. For example, if we fix $\sigma=0.5$, the fit is almost exactly the same at $\Omega=(0.163,1.968,0.5)$, the sum of squared residuals changing only at the fourth decimal point. Therefore for many of the experiments below we simply set $\sigma=0.5$ and fit $(\eta, \sigma)$. This makes $\sigma$ as big as possible, but still implies the decentralized market contributes no more than around $10 \%$ of aggregate output at a benchmark annual inflation rate of $4 \%$.

This completes the baseline calibration, except for the bargaining parameter $\theta$. We will consider three values: $\theta=1$, which eliminates the holdup problem and makes our setup more comparable to previous studies; $\theta=1 / 2$, which means symmetric bargaining; and finally, the value $\theta_{\mu}$ calibrated to generate a markup $\mu$ (price over marginal cost) consistent with the evidence. We use as a target $\mu=1.1$, which is standard in the literature following Basu and Fernald (1997). To compute the markup in the model, note that price over marginal cost in the decentralized market is $\mu_{d}=\phi M / q$, while in the centralized market it is $\mu_{c}=1$. Aggregate $\mu$ averages these using the shares of $Y$ produced in each sector. In equilibrium, $\mu$ depends on $\tau$ as well as $\theta$ 


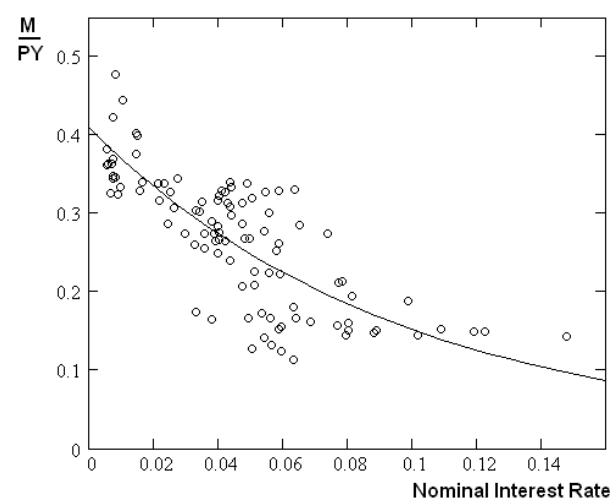

Figure 6: The "money demand" data and fitted $L(i)$.

and $\Omega$. We fit $\Omega$ and $\theta$ to the data, subject to the constraint $\mu=1.1$, at the benchmark inflation rate of $4 \%$. In what follows, then, we consider $\theta=1$, $1 / 2$, and $\theta_{\mu}$.

As is standard, our measure of the welfare cost of inflation asks how much agents would be willing to give up in terms of consumption to have inflation 0 instead of $\tau$. In steady state, for any $\tau$, write utility as

$$
(1-\beta) V(\tau)=U\left(X^{*}\right)-X^{*}+\alpha \sigma\left\{u\left[q^{s}(\tau)\right]-q^{s}(\tau)\right\}
$$

Suppose we reduce $\tau$ to 0 but also reduce consumption of both general and special goods by a factor $\Delta$, so that utility becomes

$$
(1-\beta) V_{\Delta}(0)=U\left(X^{*} \Delta\right)-X^{*}+\alpha \sigma\left\{u\left[q^{s}(0) \Delta\right]-q^{s}(0)\right\}
$$

We measure the welfare cost of $\tau$ as the value of $\Delta_{0}$ that solves $V_{\Delta}(0)=V(\tau)$; agents would give up $1-\Delta_{0}$ percent of consumption to have 0 rather than $\tau$. We also consider $\Delta_{F}$, which is how much they would give up to have the Friedman Rule $\tau^{F}$ rather than $\tau$. Our experiments below use $\tau=0.1$ (i.e., 
we consider the welfare cost of a $10 \%$ inflation) but we also report the welfare costs of inflation rates ranging from 0 to $200 \%$ at the end of the section.

In Table 1 , the first column of results is for $\theta=1$ and the fitted $\Omega$, while the second column of results keeps $\theta=1$, fixes $\sigma=0.5$ and refits $(\eta, B)$. As one can see, the welfare costs of inflation are the same, $1-\Delta_{0}=0.014$ and $1-\Delta_{F}=0.016$ (although the equilibrium $q$ is slightly different, as is the ratio of $Y$ at $\tau=0.1$ to $Y$ at $\tau=0$ and the share of the decentralized sector $\left.s_{d}\right)$. Since it makes little difference for the welfare results, for the rest of the experiments in Table 1 we fix $\sigma=0.5$ and fit only $(\eta, B)$. In any case, the numbers for our welfare cost in the case $\theta=1$ are very similar to, if perhaps slightly bigger than, the typical estimates in the literature-including Lucas (2000), who actually gives a range of numbers depending on some details, but reports the typical result for $1-\Delta_{0}$ as just under $1 \%$. We interpret our results with $\theta=1$ as being basically consistent with the consensus view in the literature. ${ }^{27}$

\footnotetext{
${ }^{27}$ To consider some other studies, in Cooley and Hansen (1989) $1-\Delta_{F}=0.0015$ when the cash-in-advance constraint is monthly and $1-\Delta_{F}=0.0052$ when it is quarterly. In Cooley and Hansen (1991), with cash and credit goods, $1-\Delta_{F}=0.0038$; by adding taxes they get it up to $1-\Delta_{F}=0.0096$. Gomme (1993) finds somewhat lower numbers in an endogenous growth model. Wu and Zhang (2000) argue that the welfare cost of inflation is larger due to monopolistic competition, and provide some more references to other studies. The point here is simply that we are in the same ballpark as most of the literature when we set $\theta=1$.
} 
Table 1: Annual Model (1900-2000)

\begin{tabular}{|c|c|c|c|c|c|}
\hline$r=0.04$ & $\theta=1$ & $\theta=1$ & $\theta=0.5$ & $\theta_{\mu}=0.343$ & $\theta=1$ \\
\hline & $\sigma=0.31$ & $\sigma=0.50$ & $\sigma=0.50$ & $\sigma=0.50$ & $\sigma=0.50$ \\
$\Omega$ & $\eta=0.27$ & $\eta=0.16$ & $\eta=0.30$ & $\eta=0.39$ & $\eta=0.39$ \\
& $B=2.13$ & $B=1.97$ & $B=1.91$ & $B=1.78$ & $B=1.78$ \\
\hline$\mu(.1)-1$ & 0.000 & 0.000 & 0.031 & 0.079 & 0.000 \\
\hline$\mu_{d}(.1)-1$ & 0.000 & 0.000 & 0.559 & 1.352 & 0.000 \\
\hline$s_{d}(.1)$ & 0.034 & 0.050 & 0.055 & 0.058 & 0.128 \\
\hline$q(\tau)$ & 0.243 & 0.206 & 0.143 & 0.094 & 0.522 \\
\hline$q(0)$ & 0.638 & 0.618 & 0.442 & 0.296 & 0.821 \\
\hline$q\left(\tau^{F}\right)$ & 1.000 & 1.000 & 0.779 & 0.568 & 1.000 \\
\hline$Y(\tau)$ & 0.947 & 0.909 & 0.914 & 0.918 & 0.932 \\
\hline$Y(0)$ & 0.014 & 0.014 & 0.032 & 0.046 & 0.012 \\
\hline $1-\Delta_{0}$ & 0.016 & 0.016 & 0.041 & 0.068 & 0.013 \\
\hline
\end{tabular}

The third column of results in Table 1 now sets $\theta=0.5$, and refits $(\eta, B)$. The fit does not suffer as a result of changing $\theta$ (the new curve lies essentially on top of the old one in Figure 6). However, the welfare costs go up considerably: $1-\Delta_{0}=0.032$ and $1-\Delta_{F}=0.041$. As one can see, $q$ is considerably below $q^{*}$ at $\tau=0.1$, and even at $\tau=0$ or $\tau=\tau^{F}$. This says the holdup problem is serious and this is what generates a relatively large cost of inflation. For this parameterization the aggregate markup is $\mu=1.04$, which comes from a markup in the decentralized market of $\mu_{d}=1.44$. The fourth column reports results with $\theta_{\mu}$ set to generate $\mu=1.10$, again refitting $(\eta, B)$. Now the costs are $1-\Delta_{0}=0.046$ and $1-\Delta_{F}=0.068$. To verify that it is the holdup problem that lies at the heart of these effects, the last column uses the same $\Omega$ as the fourth column but sets $\theta=1$. This yields costs even lower than in the first two columns. Hence, it is $\theta<1$ and not the other parameters that generates the big welfare costs. 
Table 2: Annual Model (1959-2000)

\begin{tabular}{|c|c|c|c|c|}
\hline$r=0.04$ & $\theta=1$ & $\theta=0.5$ & $\theta_{\mu}=0.404$ & $\theta=1$ \\
\hline \multirow{3}{*}{$\Omega$} & $\sigma=0.50$ & $\sigma=0.50$ & $\sigma=0.50$ & $\sigma=0.50$ \\
& $\eta=0.27$ & $\eta=0.45$ & $\eta=0.48$ & $\eta=0.48$ \\
& $B=3.19$ & $B=2.92$ & $B=2.71$ & $B=2.71$ \\
\hline$\mu(.1)-1$ & 0.000 & 0.054 & 0.084 & 0.000 \\
\hline$\mu_{d}(.1)-1$ & 0.000 & 0.910 & 1.451 & 0.000 \\
\hline$s_{d}(.1)$ & 0.058 & 0.059 & 0.058 & 0.098 \\
\hline$q(\tau)$ & 0.392 & 0.192 & 0.135 & 0.590 \\
\hline$q(0)$ & 0.752 & 0.409 & 0.307 & 0.852 \\
\hline$q\left(\tau^{F}\right)$ & 1.000 & 0.602 & 0.478 & 1.000 \\
\hline$\frac{Y(\tau)}{Y(0)}$ & 0.949 & 0.950 & 0.949 & 0.958 \\
\hline $1-\Delta_{0}$ & 0.008 & 0.025 & 0.031 & 0.007 \\
\hline $1-\Delta_{F}$ & 0.009 & 0.035 & 0.046 & 0.008 \\
\hline
\end{tabular}

Table 2 reports similar experiments fitting the model to a shorter sample. In each case we fix $\sigma=0.5$ and fit $(\eta, B)$. The qualitative conclusions are similar to what we found with the longer sample. First, with $\theta=1$ the cost of inflation is even lower than shown in Table 1, and thus even more in line with the literature, coming in at just under 1\%. Second, decreasing $\theta$ to 0.5 or to the $\theta_{\mu}$ that generates $\mu=1.1$ and then refitting $(\eta, B)$, we increase the costs considerably; e.g., given $\theta_{\mu}$, going from $\tau=0.1$ to 0 is now worth $3.1 \%$ of consumption, and going all the way to $\tau^{F}$ is now worth $4.6 \%$. While smaller than the analogous numbers in Table 1, these are still big. It is interesting (if perhaps not surprising) that the more recent data gives different "money demand" parameters and a different welfare cost, but what we want to emphasize here is that regardless of the sample period, going from $\theta=1$ to $\theta<1$ can substantially increase the estimated cost of inflation. And again, the final column makes it clear that it is the value of $\theta$, and not the values of $(\eta, B)$, that is the key to the results. 
Table 3: Monthly Model (1900-2000)

\begin{tabular}{|c|c|c|c|c|}
\hline$r=0.04$ & $\theta=1$ & $\theta=0.5$ & $\theta_{\mu}=0.315$ & $\theta=1$ \\
\hline & $\sigma=0.033$ & $\sigma=0.052$ & $\sigma=0.052$ & $\sigma=0.052$ \\
$\Omega$ & $\eta=0.20$ & $\eta=0.23$ & $\eta=0.33$ & $\eta=0.33$ \\
& $B=0.17$ & $B=0.15$ & $B=0.14$ & $B=0.14$ \\
\hline$\mu(.1)-1$ & 0.000 & 0.027 & 0.080 & 0.000 \\
\hline$\mu_{d}(.1)-1$ & 0.000 & 0.395 & 1.101 & 0.000 \\
\hline$s_{d}(.1)$ & 0.043 & 0.068 & 0.073 & 0.17 \\
\hline$q(\tau)$ & 0.230 & 0.151 & 0.101 & 0.552 \\
\hline$q(0)$ & 0.623 & 0.476 & 0.329 & 0.831 \\
\hline$q\left(\tau^{F}\right)$ & 1.000 & 0.845 & 0.644 & 1.000 \\
\hline$\frac{Y(\tau)}{Y(0)}$ & 0.932 & 0.890 & 0.894 & 0.921 \\
\hline $1-\Delta_{0}$ & 0.013 & 0.030 & 0.049 & 0.010 \\
\hline $1-\Delta_{F}$ & 0.015 & 0.038 & 0.069 & 0.011 \\
\hline
\end{tabular}

In Table 3 we recalibrate so that the period is 1 month-i.e., we simply transform $Y$ and $i$ in the data to make them monthly. The first column again fits $\Omega$ with $\theta=1$. Notice that, naturally, the estimated $\sigma$ and $B$ are lower in the monthly model, but the overall fit with the "money demand" data is about the same. In any case, what we really want to emphasize is that the costs of inflation are almost identical to those in the first two columns of Table 1. The second column in Table 3 uses $\theta=0.5$ while the third column uses $\theta_{\mu}$, and again the results are almost identical to those in the analogous cases in Table 1 . So the basic conclusion is robust to changing period length as well as the sample.

Finally, Figure 7 shows the welfare cost $1-\Delta_{0}$ for inflation rates ranging from 0 to $150 \%$; as is clear from the figure the cost basically converges by $\tau=1.5$ since decentralized trade has all but shut down at this point. The upper curve is for $\theta_{\mu}$ and the implied $\Omega$ from the fourth column of Table 1 , while the lower curve is for $\theta=1$ and the same $\Omega$. The difference in the 


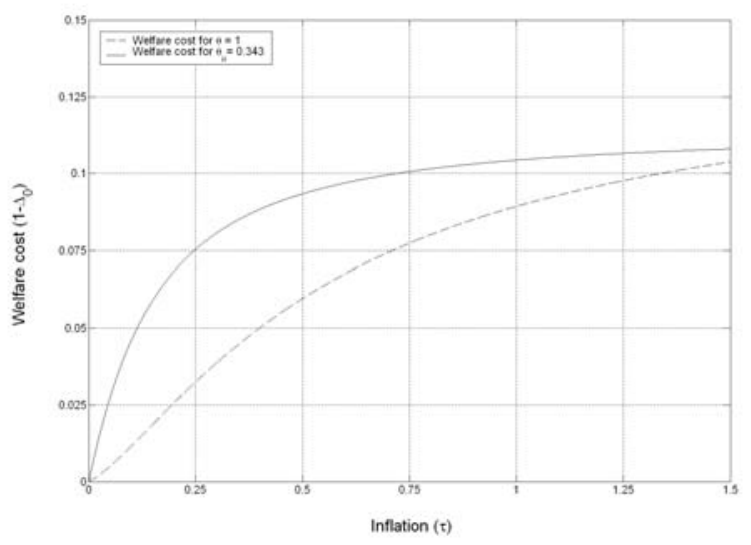

Figure 7: Welfare costs of large inflations.

curves is due to the holdup problem. Notice that the difference gets smaller at big inflation rates, because $q$ gets very small for big $\tau$ regardless of $\theta$. Hence, the real difference between models with $\theta=1$ and $\theta<1$ is for small to moderate inflation rates.

\section{Conclusion}

This paper has presented a new framework for monetary theory and policy analysis. It is based explicitly on the frictions that make money essential in the search literature, but without the extreme restrictions usually made in those models about individuals' money holdings. The key innovation here is to allow agents to interact periodically in decentralized and centralized markets. Given this, if agents have quasi-linear preferences over the good traded in the centralized market the distribution of money holdings will be degenerate in equilibrium. This makes the model very tractable. Once we have a tractable model it is easy to use it to ask many interesting questions. 
We characterized equilibria and discussed some policy issues. The Friedman Rule is optimal, but for bargaining power below 1 it does not achieve the first best. This can have important quantitative implications for the welfare cost of inflation, as we showed in a calibrated version of the model. It is not hard to generate welfare costs equivalent to $3 \%$ or $5 \%$ of consumptionmuch bigger than most existing estimates. We also showed how to extend the model to allow uncertainty in real or monetary variables. We think all of this constitutes progress in terms of bringing micro and macro models of monetary economics closer together. We also think that we have only scratched the surface, and much more could be done in terms of applications and extensions in future research. 


\section{A Appendix}

In this Appendix we first verify that the bargaining solutions are as claimed in the text. We then use these results to derive, without using value function $V$, certain properties any equilibrium must satisfy. We then use these properties to establish the existence and uniqueness of $V$. Finally, we provide some details for the proof of Proposition 1.

Lemma 1 In a double coincidence meeting each agent produces $q^{*}$ and no money changes hands.

Proof. The symmetric Nash problem is

$$
\max _{q_{1}, q_{2}, \Delta}\left[u\left(q_{1}\right)-c\left(q_{2}\right)-\phi \Delta\right]\left[u\left(q_{2}\right)-c\left(q_{1}\right)+\phi \Delta\right]
$$

subject to $-m_{2} \leq \Delta \leq m_{1}$, where $q_{1}$ and $q_{2}$ denote the quantities consumed by agents 1 and 2 and $\Delta$ is the amount of money 1 pays 2 . There is a unique solution, characterized by the first order conditions

$$
\begin{aligned}
u^{\prime}\left(q_{1}\right)\left[u\left(q_{2}\right)-c\left(q_{1}\right)+\phi \Delta\right] & =c^{\prime}\left(q_{1}\right)\left[u\left(q_{1}\right)-c\left(q_{2}\right)-\phi \Delta\right] \\
c^{\prime}\left(q_{2}\right)\left[u\left(q_{2}\right)-c\left(q_{1}\right)+\phi \Delta\right] & =u^{\prime}\left(q_{2}\right)\left[u\left(q_{1}\right)-c\left(q_{2}\right)-\phi \Delta\right] \\
u\left(q_{1}\right)-u\left(q_{2}\right)+c\left(q_{1}\right)-c\left(q_{2}\right)-2 \phi \Delta & =\frac{(2 / \phi)\left(\lambda_{1}-\lambda_{2}\right)}{\left\{\left[u\left(q_{1}\right)-c\left(q_{2}\right)-\phi \Delta\right]\left[u\left(q_{2}\right)-c\left(q_{1}\right)+\phi \Delta\right]\right\}^{-1 / 2}}
\end{aligned}
$$

where $\lambda_{i}$ is the multiplier on agent $i$ 's cash constraint. It is easy to see that $q_{1}=q_{2}=q^{*}$ and $\Delta=\lambda_{1}=\lambda_{2}=0$ solves these conditions.

Lemma 2 In a single coincidence meeting the bargaining solution is given by (6). 
Proof. The necessary and sufficient conditions for (5) are

$$
\begin{aligned}
\theta[\phi d-c(q)] u^{\prime}(q)= & (1-\theta)[u(q)-\phi d] c^{\prime}(q) \\
\theta[\phi d-c(q)] \phi= & (1-\theta)[u(q)-\phi d] \phi \\
& -\lambda[u(q)-\phi d]^{1-\theta}[\phi d-c(q)]^{\theta}
\end{aligned}
$$

where $\lambda$ is the Lagrange multiplier on $d \leq m$. There are two possible cases: If the constraint does not bind, then $\lambda=0, q=q^{*}$ and $d=m^{*}$. If the constraint binds then $q$ is given by (27) with $d=m$, which is (7).

We now present some arbitrage-style arguments to establish that any equilibrium must satisfy certain conditions. These arguments do not use any properties of $V$ or $F$.

Lemma 3 In any equilibrium, $\beta \phi_{t+1} \leq \phi_{t}$ for all $t$.

Proof. First, note that lifetime utility is finite in any equilibrium. Now suppose by way of contradiction that $\beta \phi_{t+1}>\phi_{t}$ at some $t$. In this case, an agent could raise his production of $Y_{t}$ by $d Y$ and sell it for $d Y / \phi$ dollars, then use the money at $t+1$ to reduce $Y_{t+1}$ by $d Y / \phi_{t+1}$ without changing anything else in his lifetime. Since utility is linear in $Y$, the net gain from this is $d Y\left(-1+\beta \phi_{t+1} / \phi_{t}\right)>0$. Hence $\beta \phi_{t+1}>\phi_{t}$ cannot hold in equilibrium.

Lemma 4 In any equilibrium, $m_{t+1}<m_{t+1}^{*}$ for all $t$ and for all agents.

Proof. Suppose $m_{t+1}>m_{t+1}^{*}$ for some $t$ and some agent. At $t$ he can change $Y_{t}$ by $d Y<0$ and carry $d m_{t+1}=d Y / \phi_{t}$ fewer dollars into $t+1$. Given $m_{t+1}>m_{t+1}^{*}$, for small $d Y$, the bargaining solution says this does not affect his payoff in the decentralized market. Hence, he can increase $Y_{t+1}$ by 
$d Y_{t+1}=-d Y \phi_{t+1} / \phi_{t}$ and not change anything else in his lifetime, for a net utility gain of $d Y\left(1-\beta \phi_{t+1} / \phi_{t}\right)>0$ by Lemma 3 . This proves $m_{t+1} \leq m_{t+1}^{*}$.

To establish the strict inequality, assume $m_{t+1}=m_{t+1}^{*}$. Again change $Y_{t}$ by $d Y<0$ and carry $d m_{t+1}=d Y / \phi_{t}$ fewer dollars into $t+1$. If he buys in the decentralized market next period he gets a smaller $q$ but the continuation value is the same from then on (he still spends all his money). If he is not a buyer then he can increase $Y_{t+1}$ by $d Y_{t+1}=-d Y \phi_{t+1} / \phi_{t}$ and not change anything else in his lifetime. The net expected utility gain from this is

$$
\begin{aligned}
D & =-d Y+\beta\left[\alpha \sigma u^{\prime}\left(q_{t+1}\right) \widehat{q}^{\prime}\left(m_{t+1}\right)+(1-\alpha \sigma) \phi_{t+1}\right] d Y / \phi_{t} \\
& =-d Y\left(\phi_{t}-\beta \phi_{t+1}\right) / \phi_{t}+\beta \alpha \sigma\left[u^{\prime}\left(q_{t+1}\right) \widehat{q}^{\prime}\left(m_{t+1}\right)-\phi_{t+1}\right] d Y / \phi_{t}
\end{aligned}
$$

The first term on the right-hand side is positive by Lemma 3, and the second is positive for small $d Y$ because then $m_{t+1}$ is near $m_{t+1}^{*}$ and this implies $\widehat{q}^{\prime}\left(m_{t+1}\right)<\phi_{t+1} / u^{\prime}\left(q_{t+1}\right)$ from (8).

Lemma 5 If $\theta \approx 1$ or $u^{\prime}$ is log concave then $F$ is degenerate in any monetary equilibrium: all agents have $m_{t+1}=M$. Given $F$ is degenerate, $\phi_{t}=G\left(\phi_{t+1}\right)$ for all $t$ where $G$ is a time-invariant continuous function.

Proof. Consider the following sequence problem: given any path $\left\{\phi_{t}, F_{t}\right\}$ and $m_{0}$,

$$
\max _{\left\{m_{t+1}\right\}_{t=0}^{\infty}} \sum_{t=0}^{\infty} \beta^{t}\left[v\left(m_{t}, \phi_{t}, F_{t}\right)+\phi_{t}\left(m_{t}-m_{t+1}\right)\right]
$$

where $v$ is defined in (10) (which does not use $V$ and is defined in terms date $t$ variables only). We know $v$ is $C^{n-1}$; thus, if a solution exists it satisfies the necessary conditions

$$
\beta v_{1}\left(m_{t+1}, \phi_{t+1}, F_{t+1}\right)+\beta \phi_{t+1}-\phi_{t} \leq 0,=0 \text { if } m_{t+1}>0 .
$$


We have $v_{1}(m, \phi, F)=\alpha \sigma\left[u^{\prime}(q) \widehat{q}^{\prime}(m)-\phi\right]$, since we know $m<m^{*}$, where $\widehat{q}^{\prime}(m)$ is given in $(8)$.

In any (monetary) equilibrium, at least one agent must choose $m_{t+1}>0$, and for this agent

$$
\beta v_{1}\left(m_{t+1}, \phi_{t+1}, F_{t+1}\right)+\beta \phi_{t+1}-\phi_{t}=0 \text {. }
$$

A quick calculation verifies that if $\theta \approx 1$ or $u^{\prime}$ is $\log$ concave then $v_{11}<0$, which implies (30) has a unique solution: all agents choose the same $m_{t+1}=$ $M$. Hence $F_{t+1}$ is degenerate in any monetary equilibrium. Finally, (30) implies $\phi_{t}=G\left(\phi_{t+1}\right)$, where $G$ is continuous because $v_{1}$ is.

We have established $F$ degenerate in any equilibrium, without using dynamic programming. This is a step towards constructing a simple proof that $V$ exists. However, at this point an issue arises: although we know in any equilibrium that $\phi_{t}=G\left(\phi_{t+1}\right)$, for dynamic programming purposes we would like to know $\phi_{t+1}=\Phi\left(\phi_{t}\right)$, and $G$ may not be invertible. Our strategy is to restrict attention to equilibria where $\phi_{t+1}=\Phi\left(\phi_{t}\right)$ and $\Phi$ is continuous. Obviously this includes all steady state equilibria, all possible equilibria in the case where $G$ is invertible, and many other dynamic equilibria, but it does not include all possibilities. First note that any equilibrium involves selecting an initial price $\phi_{0}$, or equivalently $q_{0}$ since we can invert $\phi_{0}=\phi\left(q_{0}\right)$ by $(7)$, and then selecting future values from the correspondence $\phi_{t+1}=G^{-1}\left(\phi_{t}\right)$. We impose only that the selection $\phi_{t+1}$ from $G^{-1}\left(\phi_{t}\right)$ cannot vary with time or the value of $\phi_{t}$.

That is, while the value $\phi_{t+1}$ obviously varies with $\phi_{t}$, the rule for choosing which branch of $G^{-1}$ from which to select $\phi_{t+1}$ is assumed to be constant. We know that this is possible for a large class of dynamic equilibria; e.g., one can always use the rule "select the lowest branch of $G^{-1}$ " and construct equilibria 
where $\phi_{t} \rightarrow 0$ from any initial $\phi_{0}$ in some interval $\left(0, \bar{\phi}_{0}\right)$. While we may not pick up all possible equilibria given our restriction, we pick up a lot. And we emphasize that the purpose of this restriction is limited: we already know that $\beta \phi_{t+1} \leq \phi_{t}$ for all $t$ and that $F$ is degenerate in any equilibrium; all we are doing here is trying to guarantee $\phi_{t+1}=\Phi\left(\phi_{t}\right)$ where $\Phi$ is continuous in order to prove the existence of the value function $V$ in order to use dynamic programming (and for steady states, there is no problem).

In any case, even given $\phi_{t+1}=\Phi\left(\phi_{t}\right)$ where $\Phi$ is continuous, we still need to bound $\phi$. We do this with $M$ constant, but the arguments are basically the same when $M$ is varying over time if we work with real balances.

Lemma 6 Assume $\sup U(X)>\bar{V} \equiv \frac{u\left(q^{*}\right)+U\left(X^{*}\right)}{1-\beta}$. Then in any equilibrium $\phi$ is bounded above by $\bar{\phi}=\bar{z} / M$, where $U(\bar{z})=\bar{V}$.

Proof. Clearly lifetime utility $V$ in any equilibrium is bounded by $\bar{V}$. Consider a candidate equilibrium with $\phi M>\bar{z}$ at some date. In the candidate equilibrium, an individual with $m=M$ would want to deviate by trading all his money for general goods since $U(\phi M)>\bar{V}$. Hence, $\phi M$ is bounded above by $\bar{z}$.

Now we can we verify the existence and uniqueness of the value function.

Lemma 7 Let $S=\mathbb{R} \times[0, \bar{\phi}]$ with $\bar{\phi}$ defined as in Lemma 6, and consider the metric space given by $\mathbb{C}=\{\hat{v}: S \rightarrow \mathbb{R} \mid \hat{v}$ is bounded and continuous $\}$ together with the sup norm, $\|\hat{v}\|=\sup |\hat{v}(m, \phi)|$. Define

$$
\mathbb{C}^{\prime}=\{\hat{V}: S \rightarrow \mathbb{R} \mid \hat{V}(m, \phi)=\hat{v}(m, \phi)+\phi m \text { for some } \hat{v} \in \mathbb{C}\} .
$$


Let $\Phi:[0, \bar{\phi}] \rightarrow[0, \bar{\phi}]$ be a continuous function, and define the operator $T: \mathbb{C}^{\prime} \rightarrow \mathbb{C}^{\prime}$ by

$$
(T \hat{V})(m, \phi)=\sup _{m_{+1}}\left\{v(m, \phi)+\phi m-\phi m_{+1}+\beta \hat{V}\left[m_{+1}, \Phi(\phi)\right]\right\}
$$

where $v(m, \phi)$ is defined in (10). Then $T$ has a unique fixed point $V \in \mathbb{C}^{\prime}$.

Proof. First we show $T: \mathbb{C}^{\prime} \rightarrow \mathbb{C}^{\prime}$. For every $\hat{V} \in \mathbb{C}^{\prime}$ we can write

$$
(T \hat{V})(m, \phi)=v(m, \phi)+\phi m+\sup _{m_{+1}} w\left[m_{+1}, \Phi(\phi)\right]
$$

where $w\left[m_{+1}, \Phi(\phi)\right]=\beta \hat{v}\left[m_{+1}, \Phi(\phi)\right]+\beta \phi m_{+1}-\phi m_{+1}$ for some $\hat{v} \in \mathbb{C}$. Since $\hat{v}$ is bounded, there exists a $\bar{m}$ such that $\beta w[0, \Phi(\phi)]>\beta w\left[m_{+1}, \Phi(\phi)\right]$ for all $m_{+1} \geq \bar{m}$. Therefore,

$$
\sup _{m_{+1}} w\left[m_{+1}, \Phi(\phi)\right]=\max _{m_{+1} \in[0, \bar{m}]} w\left[m_{+1}, \Phi(\phi)\right],
$$

and the maximum is attained. Using $w^{*}(\phi)$ to denote the solution, we have $T \hat{V}(m, \phi)=v(m, \phi)+w^{*}(\phi)+\phi m \in \mathbb{C}^{\prime}$, since $w^{*}(\phi) \in \mathbb{C}$ by the Theorem of the Maximum and $v(x, \phi) \in \mathbb{C}$ from the bargaining solution.

We now show $T$ is a contraction mapping. Define the norm $\left\|\hat{V}_{1}-\hat{V}_{2}\right\|=$ $\sup \left|\hat{v}_{1}(m, \phi)-\hat{v}_{2}(m, \phi)\right|$ and consider the metric space $\left(\mathbb{C}^{\prime},\|\cdot\|\right)$. Fix $(m, \phi) \in$ $S$. Letting $m_{+1}^{i}=\underset{m_{+1} \in[0, \bar{m}]}{\arg \max }\left\{\beta \hat{V}_{i}\left[m_{+1}, \Phi(\phi)\right]-\phi m_{+1}\right\}$, we have

$$
\begin{aligned}
T \hat{V}_{1}-T \hat{V}_{2} & =\left\{\beta \hat{V}_{1}\left[m_{+1}^{1}, \Phi(\phi)\right]-\phi m_{+1}^{1}\right\}-\left\{\beta \hat{V}_{2}\left[m_{+1}^{2}, \Phi(\phi)\right]-\phi m_{+1}^{2}\right\} \\
& \leq \beta\left|\hat{V}_{1}\left[m_{+1}^{1}, \Phi(\phi)\right]-\hat{V}_{2}\left[m_{+1}^{1}, \Phi(\phi)\right]\right| \leq \beta\left\|\hat{V}_{1}-\hat{V}_{2}\right\| .
\end{aligned}
$$

Similarly, $T \hat{V}_{2}-T \hat{V}_{1} \leq \beta\left\|\hat{V}_{1}-\hat{V}_{2}\right\|$. Hence $\left|T \hat{V}_{2}-T \hat{V}_{1}\right| \leq \beta\left\|\hat{V}_{1}-\hat{V}_{2}\right\|$. Taking the supremum over $(m, \phi)$ we have $\left\|T \hat{V}_{1}-T \hat{V}_{2}\right\| \leq \beta\left\|\hat{V}_{1}-\hat{V}_{2}\right\|$, and $T$ satisfies the definition of a contraction. 
We now argue that $\left(\mathbb{C}^{\prime}, \rho\right)$ is complete. Clearly, if $\hat{V}_{n}(m, \phi)=\hat{v}_{n}(m, \phi)+$ $\phi m$ is a Cauchy sequence in $\mathbb{C}^{\prime}$ then $\left\{\hat{v}_{n}(m, \phi)\right\}$ is a Cauchy sequence in $\mathbb{C}$. Since $(\mathbb{C},\|\cdot\|)$ is complete (see, e.g., Stokey and Lucas [1989], Theorem 3.1), $\hat{v}_{n} \rightarrow v \in \mathbb{C}$. If we set $V=v+\phi m$ it is immediate that $\hat{V}_{n} \rightarrow V \in \mathbb{C}^{\prime}$. Therefore $\left(\mathbb{C}^{\prime}, \rho\right)$ is complete. It now follows from the Contraction Mapping Theorem (see, e.g., Stokey and Lucas [1989], Theorem 3.2) that $T$ has a unique fixed point $V \in \mathbb{C}^{\prime}$.

The final thing to do is fill in some details for the proof of Proposition 1. Proof of Proposition 1: Most of what is stated follows directly from the analysis in the text, but two details need to be addressed. First, consider the uniqueness of the solution to $e(q)=1+\frac{1-\beta}{\alpha \sigma \beta}$ for a general $\theta$. This would follow if $e^{\prime}(0)<0$. Given the normalization $c(q)=q$,

$$
e(q)=\frac{\left(\theta u^{\prime}+1-\theta\right)^{2} u^{\prime}}{\left(\theta u^{\prime}+1-\theta\right) u^{\prime}-\theta(1-\theta)(u-c) u^{\prime \prime}} .
$$

Therefore $e^{\prime}$ takes the same sign as

$$
\begin{aligned}
D_{1}= & {\left[\left(\theta u^{\prime}+1-\theta\right) u^{\prime}-\theta(1-\theta)(u-c) u^{\prime \prime}\right]\left[\left(\theta u^{\prime}+1-\theta\right) u^{\prime \prime}+2 \theta u^{\prime} u^{\prime \prime}\right] } \\
& -u^{\prime}\left(\theta u^{\prime}+1-\theta\right)\left[\left(\theta u^{\prime}+1-\theta\right) u^{\prime \prime}+\theta u^{\prime} u^{\prime \prime}\right] \\
& +u^{\prime}\left(\theta u^{\prime}+1-\theta\right)\left[\theta(1-\theta)\left(u^{\prime}-1\right) u^{\prime \prime}+\theta(1-\theta)(u-c) u^{\prime \prime \prime}\right] .
\end{aligned}
$$

After simplification we arrive at

$$
\begin{aligned}
D_{1}= & -2 \theta^{2}(1-\theta)(u-c) u^{\prime} u^{\prime 2}+\theta\left(\theta u^{\prime}+1-\theta\right)\left[u^{\prime}+(1-\theta)\left(u^{\prime}-1\right) u^{\prime} u^{\prime \prime}\right] \\
& -\theta(1-\theta)\left(\theta u^{\prime}+1-\theta\right)(u-c) u^{\prime \prime 2}+\theta(1-\theta)\left(\theta u^{\prime}+1-\theta\right)(u-c) u^{\prime} u^{\prime \prime \prime} .
\end{aligned}
$$

Since $q^{s}<q^{*}$, we have $u^{\prime}>1$ and all but the final term are unambiguously negative. If $\theta=1$ this term vanishes and $D_{1}<0$. For any $\theta \in(0,1)$, if $u^{\prime}$ is 
log-concave then $u^{\prime} u^{\prime \prime \prime}<u^{\prime \prime}$, and this term is bounded by the previous term and so again we have $D_{1}<0$.

Second, we show that $e$ shifts up with an increase in $\theta$ at the solution to $e(q)=1+\frac{1-\beta}{\alpha \sigma \beta}$. To begin, note that $\partial e / \partial \theta$ takes the same sign as

$$
\begin{aligned}
D_{2}= & 2\left(u^{\prime}-1\right)\left[\left(\theta u^{\prime}+1-\theta\right) u^{\prime}-\theta(1-\theta)(u-c) u^{\prime \prime}\right] \\
& -\left(\theta u^{\prime}+1-\theta\right)\left[u^{\prime}\left(u^{\prime}-1\right)-(1-2 \theta)(u-c) u^{\prime \prime}\right] \\
= & \left(\theta u^{\prime}+1-\theta\right)\left(u^{\prime}-1\right) u^{\prime}-\left(\theta u^{\prime}-1+\theta\right)(u-c) u^{\prime \prime}
\end{aligned}
$$

Now rearrange $e(q)=1+\frac{1-\beta}{\alpha \sigma \beta}$ as

$$
(u-c) u^{\prime \prime}=\frac{\left(\theta u^{\prime}+1-\theta\right) u^{\prime}}{\theta(1-\theta)\left(1+\frac{1-\beta}{\alpha \sigma \beta}\right)}\left(\frac{1-\beta}{\alpha \sigma \beta}+\theta-\theta u^{\prime}\right)
$$

Substituting this into $D_{2}$ and simplifying, at $e(q)=1+\frac{1-\beta}{\alpha \sigma \beta}$ we see that $D_{2}$ takes the same sign as $\frac{1-\beta}{\alpha \sigma \beta}(1-\theta)^{2}+\theta^{2} u^{\prime}\left(u^{\prime}-e\right)$. The desired result follows if we can show $u^{\prime} \geq e$, which is easy to establish. 


\section{References}

[1] Aiyagari, S. Rao, and Neil Wallace. "Existence of Steady States with Positive Consumption in the Kiyotaki-Wright Model." Review of Economic Studies 58(5) (October 1991): 901-916.

[2] Alvarez, Fernando, and Nancy L. Stokey. "Dynamic Programming with Homogeneous Functions." Journal of Economic Theory 82(1) (September 1998): 167-189.

[3] Aruoba, S. Boragan and Randall Wright. "Search, Money, and Capital: A Neoclassical Dichotomy." Unpublished manuscript, Federal Reserve Bank of Cleveland, 2002.

[4] Basu, Susanto and Fernald, John. "Returns to scale in U.S. production: Estimates and implications," Journal of Political Economy 105(2) (April 1997): 249-283.

[5] Berentsen, Aleksander. "On the Distribution of Money Holdings in a Random-Matching Model." International Economic Review 43(3) (August 2002): 945-954.

[6] Berentsen, Aleksander, and Guillaume Rocheteau. "Money and the Gains from Trade." International Economic Review 44(1) (February 2003): 263-297.

[7] Berentsen, Aleksander, and Guillaume Rocheteau. "On the Efficiency of Monetary Exchange: How Divisibility of Money Matters." Journal of Monetary Economics 49(8) (November 2002): 1621-1649.

[8] Berentsen, Aleksander, and Guillaume Rocheteau. "On the Friedman Rule in Search Models with Divisible Money." Contributions to Macroeconomics 3(1) 2003.

[9] Berentsen, Aleksander, Guillaume Rocheteau and Shouyong Shi. "Friedman Meets Hosios: Efficiency in Search Models of Money." Unpublished manuscript, 2001.

[10] Camera, Gabriele, and Dean Corbae. "Money and Price Dispersion." International Economic Review 40(4) (November 1999): 985-1008. 
[11] Christiano, Lawrence, Martin Eichenbaum. "Current Real-BusinessCycle Theories and Aggregate Labor-Market Fluctuations," American Economic Review 82(3) (June 1992): 430-450.

[12] Christiano, Lawrence, Martin Eichenbaum and Charles Evans. "Sticky Price and Limited Participation Models: A Comparison," European Economic Review 41(6) (June 1997): 1201-1249.

[13] Cooley, Thomas and Gary Hansen. "The Inflation Tax in a Real Business Cycle Model." American Economic Review 79(4) (September 1989): 733-748.

[14] Cooley, Thomas and Gary Hansen. "The Welfare Costs of Moderate Inflations." Journal of Money, Credit and Banking 23(3) (August 1991, Part 2): 483-503.

[15] Duffie, Darrell, John Geanakoplos, Andreu Mas-Colell and Andrew McLennan. "Stationary Markov Equilibria." Econometrica 62(4) (July 1994): 745-781.

[16] Faig, Miguel. "A Search Theory of Money with Commerce and Neoclassical Production." Unpublished manuscript, 2001.

[17] Friedman, Milton. The Optimal Quantity of Money and Other Essays. Chicago: Adeline, 1969.

[18] Gomme, Paul. "Money and Growth Revisited: Measuring the Cost of Inflation in an Endogenous Growth Model." Journal of Monetary Economics 32(1) (August 1993): 51-77.

[19] Green, Edward, and Ruilin Zhou. "A Rudimentary Random-Matching Model with Divisible Money and Prices." Journal of Economic Theory 81(2) (August 1998): 252-271.

[20] Hansen, Gary D. "Indivisible Labor and the Business Cycle," Journal of Monetary Economics 16(3) (November 1985): 309-327.

[21] Head, Allen and Alok Kumar. "Price-posting, price dispersion, and inflation in a random matching model." International Economic Review (2005), in press. 
[22] Hosios, Arthur J. "On the Efficiency of Matching and Related Models of Search and Unemployment." Review of Economic Studies 57(2) (April 1990): 279-298.

[23] Khan, Aubhik, Julia K. Thomas, and Randall Wright. "Money and Distribution: Quantitative Theory and Political Analysis." Unpublished manuscript, University of Pennsylvania, 2003.

[24] Kiyotaki, Nobuhiro and John Moore. "Liquidity, Business Cycles and Monetary Policy." Lecture 2, Clarendon Lectures, November 2001.

[25] Kiyotaki, Nobuhiro and Randall Wright. "On Money as a Medium of Exchange." Journal of Political Economy 97(4) (August 1989): 927-954.

[26] Kiyotaki, Nobuhiro and Randall Wright. "A Contribution to the Pure Theory of Money." Journal of Economic Theory 53(2) (April 1991): 215-235.

[27] Kiyotaki, Nobuhiro and Randall Wright. "A Search-Theoretic Approach to Monetary Economics." American Economic Review 83(1) (March 1993): 63-77.

[28] Kocherlakota, Narayana. "Money is Memory." Journal of Economic Theory 81(2) (August 1998): 232-251.

[29] Lagos, Ricardo and Randall Wright "Dynamics, Cycles and Sunspot Equilibria in 'Genuinely Dynamic, Fundamentally Disaggregative' Models of Money." Journal of Economic Theory 109(2) (April 2003): 156171.

[30] Lagos, Ricardo and Guillaume Rocheteau. "Coexistence of Money and Real Assets." Unpublished manuscript, 2002.

[31] Lucas, Robert E. Jr. "Inflation and Welfare." Econometrica 68(2) (March 2000): 247-274.

[32] Lucas, Robert E. Jr. and Nancy Stokey. "Optimal Fiscal and Monetary Policy in an Economy without Capital." Journal of Monetary Economics 12(1) (July 1983): 55-93. 
[33] Lucas, Robert E. Jr. and Nancy Stokey. "Money and Interest in a Cashin-Advance Economy." Econometrica 55(3) (May 1987): 491-513.

[34] Molico, Miguel. "The Distribution of Money and Prices in Search Equilibrium." unpublished manuscript, 1999.

[35] Rauch, Bernard. "A Divisible Search Model of Fiat Money: A Comment." Econometrica 68(1) (January 2000): 149-156.

[36] Rogerson, Richard. "Indivisible Labor, Lotteries and Equilibrium." Journal of Monetary Economics 21(1) (1988): 3-16.

[37] Shi, Shouyong. "Money and Prices: A Model of Search and Bargaining." Journal of Economic Theory 67(2) (December 1995): 467-496.

[38] Shi, Shouyong. "A Divisible Search Model of Fiat Money." Econometrica 64(1) (January 1997): 75-102.

[39] Shi, Shouyong. "Search for a Monetary Propagation Mechanism." Journal of Economic Theory 81(2) (August 1998): 314-352.

[40] Shi, Shouyong. "Search, Inflation and Capital Accumulation." Journal of Monetary Economics 44(1) (August 1999): 81-103.

[41] Stokey, Nancy and Lucas, Robert E. Jr. Recursive Methods in Economic Dynamics. Cambridge: Harvard University Press, 1989.

[42] Taber, Alexander and Neil Wallace. "A Matching Model with Bounded Holdings of Indivisible Money." International Economic Review 40(4) (November 1999): 961-984.

[43] Trejos, Alberto and Randall Wright. "Search, Bargaining, Money and Prices." Journal of Political Economy 103(1) (February 1995): 118-141.

[44] Walsh, Carl E. Monetary Theory and Policy. Cambridge and London: MIT Press, 1998.

[45] Wallace, Neil. "Whither monetary economics?" International Economic Review 42(4) (November 2001): 847-869. 
[46] Williamson, Steve and Wright, Randall (2003). "Seasonality and monetary policy." Unpublished manuscript, 2003.

[47] Wu, Yangru and Junxi Zhang. "Monopolistic Competition, Increasing Returns to Scale and the Welfare Costs of Inflation." Journal of Monetary Economics 46(2) (October 2000): 417-440.

[48] Zhou, Ruilin. "Individual and Aggregate Real Balances in a RandomMatching Model." International Economic Review 40(4) (November 1999): 1009-1038. 